ESAIM: M2AN 46 (2012) 317-339

DOI: $10.1051 / \mathrm{m} 2 \mathrm{an} / 2011045$
ESAIM: Mathematical Modelling and Numerical Analysis

www.esaim-m2an.org

\title{
ON THE CONVERGENCE OF GENERALIZED POLYNOMIAL CHAOS EXPANSIONS *
}

\author{
Oliver G. Ernst ${ }^{1}$, Antje Mugler ${ }^{2}$, Hans-Jörg StarklofF ${ }^{2}$ \\ AND Elisabeth UlLMANN ${ }^{1}$
}

\begin{abstract}
A number of approaches for discretizing partial differential equations with random data are based on generalized polynomial chaos expansions of random variables. These constitute generalizations of the polynomial chaos expansions introduced by Norbert Wiener to expansions in polynomials orthogonal with respect to non-Gaussian probability measures. We present conditions on such measures which imply mean-square convergence of generalized polynomial chaos expansions to the correct limit and complement these with illustrative examples.
\end{abstract}

Mathematics Subject Classification. 33C45, 35R60, 40A30, 41A10, 60H35, 65N30.

Received January 18, 2011

Published online October 12, 2011.

\section{INTRODUCTION}

A fundamental task in computational stochastics is the accurate representation of random quantities such as random variables, stochastic processes and random fields using a manageable number of degrees of freedom. A popular approach, known by the names polynomial chaos expansion, Wiener-Hermite expansion or FourierHermite expansion, represents a random variable by a series of Hermite polynomials in a countable sequence of independent Gaussian random variables - so-called basic random variables - , and employs truncations of such expansions as approximations. While the origins of this approach date back to the 1930s, renewed interest in Wiener-Hermite expansions has resulted from recent developments in computational methods for solving stochastic partial differential equations (SPDEs), specifically partial differential equations with random data $[2,3,14,27,42,46]$. Solutions of such equations are stochastic processes indexed by time and/or spatial coordinates, and in the latter case are referred to as random fields. A pivotal contribution in this context is the work of Ghanem and Spanos [14], who proposed using truncated polynomial chaos expansions as trial functions in a Galerkin framework, resulting in their spectral stochastic finite element method, now commonly known as the stochastic Galerkin method.

Keywords and phrases. Equations with random data, polynomial chaos, generalized polynomial chaos, Wiener-Hermite expansion, Wiener integral, determinate measure, moment problem, stochastic Galerkin method, spectral elements.

* This work was supported by the Deutsche Forschungsgemeinschaft Priority Programme 1324.

1 Institut für Numerische Mathematik und Optimierung, TU Bergakademie Freiberg, 09596 Freiberg, Germany. ernst@math.tu-freiberg.de; ullmann@math.tu-freiberg.de

2 Fachgruppe Mathematik, University of Applied Sciences Zwickau, 08012 Zwickau, Germany. Antje.Mugler@fh-zwickau.de; hans.joerg.starkloff@fh-zwickau.de 
A fundamental result of Cameron and Martin [7] states that polynomials in a countable sequence of independent standard Gaussian random variables lie dense in the set of random variables with finite variance which are measurable with respect to these Gaussian random variables. However, the number of random variables and the polynomial degree required for a sufficient approximation depend on the functional dependence of this random variable on the Gaussian random variables. In a series of papers [22, 47-50,52], Xiu and Karniadakis discovered that better approximation of random variables can often be achieved using polynomial expansions in non-Gaussian basic random variables, which they termed generalized polynomial chaos expansions. To retain the convenience of working with orthogonal polynomials, in generalized polynomial chaos expansions the Hermite polynomials are replaced by the sequence of polynomials orthogonal with respect to the probability distribution of the basic random variables.

With regard to the convergence of these generalized expansions Xiu and Karniadakis remark in one of their earlier papers on this topic [47], page 4930: "convergence to second-order stochastic processes can be possibly obtained as a generalization of Cameron-Martin theorem". However, the question of convergence has been approached rather guardedly even in very recent publications on the subject such as Arnst et al. [1], page 3137, who write: "however, it should be stressed that, in the present state of the art in mathematics, the convergence of a chaos expansion for a second-order random variable with values in an infinite-dimensional space can be obtained only if the germ is Gaussian".

The open question we answer in the present work is precisely under which conditions the convergence of polynomial chaos expansions carries over to generalized polynomial chaos expansions. We show, based on classical results on the Hamburger moment problem, that an arbitrary random variable with finite variance can only be expanded in generalized polynomial chaos if the underlying probability measure is uniquely determined by its moments. Earlier work by Segal [35] contains a first generalization of the Cameron-Martin theorem under the stronger assumption that the underlying probability distributions possess a finite moment-generating function. In other related work, Soize and Ghanem [38] have considered generalized (not necessarily polynomial) chaos expansions in a finite number of random variables, but, in contrast to the present work, density was assumed for the individual basic random variables and the infinite-dimensional case was not treated.

We also include a number of examples to emphasize that non-convergence of generalized polynomial chaos expansions can occur for relatively straightforward situations. Since stochastic Galerkin computations are currently the primary application of generalized polynomial chaos expansions we also include an example where generalized polynomial chaos expansion displays superior approximation properties over standard polynomial chaos in this method.

The plan of the remainder of this paper is as follows: Section 2 recalls basic notation, definitions and convergence results of standard Wiener-Hermite polynomial chaos expansions, including the celebrated CameronMartin theorem. Section 3 then treats generalized polynomial chaos expansions, with separate discussions of expansions in one, a finite number and a countably infinite number of basic random variables. A number of illustrative examples follow in Section 4, and various technical issues are provided in the appendix.

\section{WiENER-HERMite POLYNOMIAL CHAOS EXPANSIONS}

In this section we recall the convergence theory of standard Wiener-Hermite polynomial chaos expansions. We begin with some remarks on the origins of the basic concepts, which date back to the beginnings of modern probability theory.

\subsection{Origins}

The term polynomial chaos was originally introduced by Wiener in his 1938 paper [45], in which he applies his generalized harmonic analysis $(c f .[30,44])$ and what are now known as multiple Wiener integrals to a mathematical formulation of statistical mechanics. In that work, Wiener began with the concept of a continuous 
homogeneous chaos, which in modern terminology ${ }^{3}$ corresponds roughly to a homogeneous random field defined on $\mathbb{R}^{d}$ which, when integrated over Borel sets, yields a stationary random measure. Essentially a mathematical description of multidimensional Brownian motion, Wiener's homogeneous chaos was a generalization to what Wiener called "pure one-dimensional chaos", the random measure given by, in modern terminology, the increments of the Wiener process. The term polynomial chaos was introduced in [45] as the set of all multiple integrals taken with respect to the Wiener process, and it was shown that these form a dense subset in the homogeneous chaos. Subsequently, Cameron and Martin [7] showed that any square-integrable functional (with respect to Wiener measure) on the set of continuous functions on the interval $[0,1]$ vanishing at zero could be expanded in an $\mathrm{L}^{2}$-convergent series of Hermite polynomials in a countable sequence of Gaussian random variables. The connection between multiple Wiener integrals and Fourier-Hermite expansion is also given in [17]. A modern exposition of Hermite expansions of functionals of Brownian motion can be found e.g. in [16, 18,20]. The gestation of Wiener's work on polynomial chaos is described in [25] and additional articles in the same Wiener memorial issue of the Bulletin of the AMS, and more comprehensively in the biography [26].

In stochastic analysis there are three basic representations for square-integrable functionals of Brownian motion:

- polynomial chaos expansions;

- mean-square convergent expansions with multiple Wiener integrals; and

- stochastic Itô integrals.

There exist deep connections between these representations and each can be converted to the others. Polynomial chaos is less frequently used in this area, as Itô integrals are often more convenient, e.g., in the study of differential equations driven by the Wiener process. Also, the term polynomial chaos is sometimes replaced by Wiener-Hermite expansion to avoid confusion with the more familiar notion of chaos as it arises in the context of dynamical systems. However, polynomial chaos has received renewed attention since the work of Ghanem and Spanos [14] on stochastic finite element methods, in which random variables as well as random fields representing inputs and solutions of partial differential equations with random data are represented as Fourier-Hermite series in Gaussian random variables.

\subsection{Setting and notation}

Given a probability space $(\Omega, \mathfrak{A}, P)$, where $\Omega$ is the abstract set of elementary events, $\mathfrak{A}$ a $\sigma$-algebra of subsets of $\Omega$ and $P$ a probability measure on $\mathfrak{A}$, we assume this space to be sufficiently rich $^{4}$ that it admits the definition of nontrivial normally distributed random variables $\xi: \Omega \rightarrow \mathbb{R}$, and we denote such random variables with mean zero and variance $\sigma^{2}>0$ by $\xi \sim N\left(0, \sigma^{2}\right)$. The mean or expectation of a (not necessarily normally distributed) random variable $\xi$ will be denoted by $\langle\xi\rangle$. The Hilbert space of (equivalence classes of) real-valued random variables defined on $(\Omega, \mathfrak{A}, P)$ with finite second moments is denoted by $\mathrm{L}^{2}(\Omega, \mathfrak{A}, P)$, with inner product $(\cdot, \cdot)_{\mathrm{L}^{2}}$ and norm $\|\cdot\|_{\mathrm{L}^{2}}$. We refer to convergence with respect to $\|\cdot\|_{\mathrm{L}^{2}}$ as mean-square convergence. We shall refer to a linear subspace of $\mathrm{L}^{2}(\Omega, \mathfrak{A}, P)$ consisting of centered (i.e., with mean zero) Gaussian random variables as a Gaussian linear space and, when this space is complete, as a Gaussian Hilbert space. We emphasize that a Gaussian Hilbert space cannot contain all Gaussian random variables on the underlying probability space (see e.g. [39] for a counterexample).

\subsection{The Cameron-Martin theorem}

Since Gaussian random variables possess moments of all orders and mixed moments of independent Gaussian random variables are simply the products of the corresponding individual moments, it is easily seen that, for

\footnotetext{
${ }^{3}$ One should note that a number of basic probabilistic concepts in Wiener's work, $c f$. also [43], were developed prior to the solid foundation of probability theory provided by Kolmogorov [23].

${ }^{4}$ Otherwise there exist only trivial Gaussian random variables taking the value zero with probability one, allowing only the modeling of deterministic phenomena.
} 
any Gaussian linear space $\mathscr{H}$ and $n \in \mathbb{N}_{0}$, the set

$$
\begin{gathered}
\mathscr{P}_{n}(\mathscr{H}):=\left\{p\left(\xi_{1}, \ldots, \xi_{M}\right): p \text { is an } M \text {-variate polynomial of degree } \leq n,\right. \\
\left.\xi_{j} \in \mathscr{H}, j=1, \ldots, M, M \in \mathbb{N}\right\}
\end{gathered}
$$

is a linear subspace of $\mathrm{L}^{2}(\Omega, \mathfrak{A}, P)$, as is its closure $\overline{\mathscr{P}}_{n}(\mathscr{H})$. Note that $\mathscr{P}_{n}(\mathscr{H})$ consists of polynomials in an arbitrary number of random variables, which can be chosen arbitrarily from $\mathscr{H}$. The space $\mathscr{P}_{0}(\mathscr{H})=\overline{\mathscr{P}}_{0}(\mathscr{H})$ consists of almost surely (a.s.) constant, i.e., degenerate, random variables. Furthermore, all elements of $\mathscr{P}_{1}(\mathscr{H})$ and $\overline{\mathscr{P}}_{1}(\mathscr{H})$ are normally distributed, whereas for $n>1$ the spaces $\mathscr{P}_{n}(\mathscr{H})$ and $\overline{\mathscr{P}}_{n}(\mathscr{H})$ contain also random variables with non-Gaussian distributions. Moreover, one can show that the spaces $\mathscr{P}_{n}(\mathscr{H})$ as well as $\overline{\mathscr{P}}_{n}(\mathscr{H})$ are distinct for different values of $n$, so that in particular $\left\{\overline{\mathscr{P}}_{n}(\mathscr{H})\right\}_{n \in \mathbb{N}_{0}}$ forms a strictly increasing sequence of subspaces of $\mathrm{L}^{2}(\Omega, \mathfrak{A}, P)$. Taking orthogonal sections, we define the spaces

$$
\mathscr{H}_{n}:=\overline{\mathscr{P}}_{n}(\mathscr{H}) \cap \mathscr{P}_{n-1}(\mathscr{H})^{\perp}, \quad n \in \mathbb{N},
$$

so that, setting also $\mathscr{H}_{0}:=\mathscr{P}_{0}(\mathscr{H})=\overline{\mathscr{P}}_{0}(\mathscr{H})$, we have the orthogonal decomposition

$$
\overline{\mathscr{P}}_{n}(\mathscr{H})=\bigoplus_{k=0}^{n} \mathscr{H}_{k}
$$

where we have used $\oplus$ to denote the orthogonal sum of linear spaces. We also consider the full space

$$
\bigoplus_{n=0}^{\infty} \mathscr{H}_{n}:=\overline{\bigcup_{n=0}^{\infty} \mathscr{P}_{n}(\mathscr{H})} .
$$

Finally, we denote by $\sigma(S)$ the $\sigma$-algebra generated by a set $S$ of random variables. Note that for a Gaussian linear space $\mathscr{H}$ defined on $(\Omega, \mathfrak{A}, P)$ we always have $\sigma(\mathscr{H}) \subset \mathfrak{A}$.

The simplest nontrivial case of a one-dimensional Gaussian Hilbert space is one spanned by a single random variable $\xi \sim N(0,1)$. In this case each linear space $\mathscr{H}_{n}$ is also one-dimensional and is spanned by the Hermite polynomial of exact degree $n$ in $\xi$.

With this notation we can state the basic density theorem for polynomials of Gaussian random variables due originally to Cameron and Martin in 1947 [7]. We state the result in a somewhat more general ${ }^{5}$ form than the original, essentially following [18], where also a proof can be found.

Theorem 2.1 (Cameron-Martin theorem). In terms of the notation introduced above, the spaces $\left\{\mathscr{H}_{n}\right\}_{n \in \mathbb{N}_{0}}$ form a sequence of closed, pairwise orthogonal linear subspaces of $\mathrm{L}^{2}(\Omega, \mathfrak{A}, P)$ such that

$$
\bigoplus_{n=0}^{\infty} \mathscr{H}_{n}=\mathrm{L}^{2}(\Omega, \sigma(\mathscr{H}), P) .
$$

In particular, if $\sigma(\mathscr{H})=\mathfrak{A}$, then $\mathrm{L}^{2}(\Omega, \mathfrak{A}, P)$ admits the orthogonal decomposition

$$
\mathrm{L}^{2}(\Omega, \mathfrak{A}, P)=\bigoplus_{n=0}^{\infty} \mathscr{H}_{n} .
$$

Before proceeding to chaos expansions, we wish to point out a number of subtleties associated with the Cameron-Martin theorem. First, the elements of the spaces $\mathrm{L}^{2}$, and hence also those of $\mathscr{H}$, are equivalence

\footnotetext{
${ }^{5}$ Cameron and Martin considered the specific probability space $\Omega=\{x \in C[0,1], x(0)=0\}$, together with its Borel $\sigma$-algebra and $P$ the Wiener measure. The associated Gaussian Hilbert space $\mathscr{H}$ is then generated by Gaussian random variables corresponding to the evaluation of a function $x$ at some $t \in[0,1]$.
} 
classes of random variables. Therefore the notation $\sigma(\mathscr{H})$ implies that all such equivalent functions must be measurable, i.e., this $\sigma$-algebra is generated by one representative from each equivalence class and the events with probability zero. This remark applies also to similar situations below. In particular, all statements and equalities are understood to hold almost surely, i.e., except for possibly sets of measure zero.

Second, we emphasize that the condition $\mathfrak{A}=\sigma(\mathscr{H})$ is necessary. This follows from basic measurability properties; a relevant result is the Doob-Dynkin lemma (see e.g. [19], Lem. 1.13). A simple example where this condition is violated and the conclusion of the theorem is false can be given as follows: consider a probability space on which two independent, non-degenerate, centered random variables $\xi$ and $\eta$ are defined, where $\xi \sim$ $N(0,1)$ and $\eta$ has an arbitrary distribution with finite second moment. If $\mathscr{H}=\{c \xi: c \in \mathbb{R}\}$ denotes the one-dimensional Gaussian Hilbert space generated by $\xi$, then all projections of $\eta$ on the spaces $\mathscr{H}_{n}$ are almost surely constant with value zero, and the approximation error equals the variance of the random variable $\eta$. For another simple example where the probability space is too coarse, consider the probability space $\Omega=\mathbb{R}$ with $\sigma$-algebra $\mathfrak{A}=\sigma(\{0\},\{1\}), P(\{0\})=p, P(\{1\})=1-p, 0<p<1$. In this case the only nonempty Gaussian Hilbert space associated with this probability space is the trivial one consisting of only the equivalence class of random variables which are a.s. constant with value zero. For any random variable $\xi_{0}$ from this equivalence class there holds $\xi_{0}(0)=\xi_{0}(1)=0, \xi_{0}(\omega)=x_{0} \in \mathbb{R}$ for $\omega \notin\{0,1\}$. The corresponding generated $\sigma$-algebra $\sigma(\mathscr{H})=\sigma\left(\xi_{0}\right)$ consists only of events with probability 0 or 1 , and hence $\sigma(\mathscr{H})=\{\emptyset,\{0,1\}, \mathbb{R} \backslash\{0,1\}, \mathbb{R}\}$ and only degenerate random variables can be approximated by polynomials in "Gaussian" random variables. Nevertheless on the probability space $(\Omega, \mathfrak{A}, P)$ there exist non-degenerate random variables with finite second order moments, e.g. the random variable $\xi$ with $\xi(0)=0, \xi(1)=1$ and $\xi(\omega)=2$ otherwise, which follows a Bernoulli distribution with parameter $p$. Completion of this probability space does not change the situation.

\subsection{Chaos expansions}

For a Gaussian linear space $\mathscr{H}$, we denote by $P_{k}: \mathrm{L}^{2}(\Omega, \mathfrak{A}, P) \rightarrow \mathscr{H}_{k}$ the orthogonal projection onto $\mathscr{H}_{k}$. The Wiener-Hermite polynomial chaos expansion of a random variable $\eta \in \mathrm{L}^{2}(\Omega, \sigma(\mathscr{H}), P)$

$$
\eta=\sum_{k=0}^{\infty} P_{k} \eta
$$

thus converges in the mean-square sense and may be approximated by the partial sums

$$
\eta \approx \eta_{n}:=\sum_{k=0}^{n} P_{k} \eta .
$$

We note that the expansion (2.1) is mean-square convergent also when $\mathfrak{A} \supsetneq \sigma(\mathscr{H})$, in which case the limit is the orthogonal projection of $\eta$ onto the closed subspace $\mathrm{L}^{2}(\Omega, \sigma(\mathscr{H}), P)$.

In applications of Wiener-Hermite polynomial chaos expansions the underlying Gaussian Hilbert space is often taken to be the space spanned by a given fixed sequence $\left\{\xi_{m}\right\}_{m \in \mathbb{N}}$ of independent Gaussian random variables $\xi_{m} \sim N(0,1)$, which we shall refer to as the basic random variables. For computational purposes the countable sequence $\left\{\xi_{m}\right\}_{m \in \mathbb{N}}$ is restricted to a finite number $M \in \mathbb{N}$ of random variables. Denoting by $\mathscr{P}_{n}^{M}=$ $\mathscr{P}_{n}^{M}\left(\xi_{1}, \ldots, \xi_{M}\right)$ the space of $M$-variate polynomials of (total) degree $n$ in the random variables $\xi_{1}, \ldots, \xi_{M}$, there holds that, for any random variable $\eta \in \mathrm{L}^{2}\left(\Omega, \sigma\left(\left\{\xi_{m}\right\}_{m \in \mathbb{N}}\right), P\right)$, the approximations

$$
\eta_{n}^{M}:=P_{n}^{M} \eta \stackrel{n, M \rightarrow \infty}{\longrightarrow} \eta
$$

where $P_{n}^{M}$ denotes the orthogonal projection onto $\mathscr{P}_{n}^{M}$, converge in the mean-square sense. This follows, e.g., from the proof of Theorem 1 in [18].

It should be emphasized that the Wiener-Hermite polynomial chaos expansion converges for quite general random variables, provided their second moment is finite. In particular, their distributions can be discrete, 
singularly continuous, absolutely continuous as well as of mixed type. Moreover, it can be shown that for a nontrivial Gaussian linear space $\mathscr{H}$ and a distribution function with finite second moments there exist random variables in $\mathrm{L}^{2}(\Omega, \sigma(\mathscr{H}), P)$ possessing this distribution function (cf. e.g. [39]). In particular, Wiener-Hermite polynomial chaos expansions are possible also for random variables which are not absolutely continuous. By contrast, note that all partial sums of a Wiener-Hermite expansion are either absolutely continuous or a.s. constant.

The following theorem collects further known (cf., e.g., $[19,36])$ and practically useful results on WienerHermite polynomial chaos expansions. The statements are formulated for the approximations $\eta_{n}$, but they also hold for the approximations $\eta_{n}^{M}$.

Theorem 2.2. Under the assumptions of the Cameron-Martin theorem (Thm. 2.1), the following statements hold for the Wiener-Hermite polynomial chaos approximations

$$
\eta_{n}=\sum_{k=0}^{n} P_{k} \eta, \quad n \in \mathbb{N}_{0}
$$

of a random variable $\eta \in \mathrm{L}^{2}(\Omega, \sigma(\mathscr{H}), P)$ with respect to a Gaussian Hilbert space $\mathscr{H}$ :

(i) $\eta_{n} \stackrel{n \rightarrow \infty}{\longrightarrow} \eta$ in $L^{p}(\Omega, \sigma(\mathscr{H}), P)$ for all $0<p \leq 2$.

(ii) Relative moments converge, when they exist, i.e., for $0<p \leq 2$ there holds

$$
\lim _{n \rightarrow \infty} \frac{\left\langle\left|\eta_{n}-\eta\right|^{p}\right\rangle}{\left\langle\eta^{p}\right\rangle}=\lim _{n \rightarrow \infty} \frac{\left\langle\left|\eta_{n}-\eta\right|^{p}\right\rangle}{\left\langle|\eta|^{p}\right\rangle}=0
$$

if $\left\langle\eta^{p}\right\rangle \neq 0$ and $\left\langle|\eta|^{p}\right\rangle \neq 0$, respectively.

(iii) $\eta_{n} \rightarrow \eta$ in probability.

(iv) There is a subsequence $\left\{n_{k}\right\}_{k \in \mathbb{N}}$ with $\lim _{k \rightarrow \infty} n_{k}=\infty$ such that $\eta_{n_{k}} \rightarrow \eta$ almost surely.

(v) $\eta_{n} \rightarrow \eta$ in distribution. This implies that the associated distribution functions converge, i.e., that

$$
P\left(\eta_{n} \leq x\right)=: F_{\eta_{n}}(x) \stackrel{n \rightarrow \infty}{\longrightarrow} F_{\eta}(x):=P(\eta \leq x)
$$

at all points $x \in \mathbb{R}$ where $F_{\eta}$ is continuous. If the distribution function $F_{\eta}$ is continuous on $\mathbb{R}$ then the distribution functions converge uniformly.

(vi) The previous property implies that the quantiles of the random variables $\eta_{n}$ converge for $n \rightarrow \infty$ to the corresponding quantiles of $\eta$. (These can be set-valued).

We remark that it may also be of interest to approximate statistical quantities other than distribution functions and moments, such as probability densities (see e.g. $[10,11]$ ). In addition, other types of convergence may be relevant.

\section{Generalized polynomial Chaos expansions}

Many stochastic problems involve non-Gaussian random variables. When these are approximated with Wiener-Hermite polynomial chaos expansions it is often observed that these expansions converge very slowly. The reason for this is that, when expressed as functions of a collection of Gaussian basic random variables, these functions are often highly nonlinear and can only be well approximated by truncated Wiener-Hermite expansions of very high order. A possible remedy is to base the expansion on non-Gaussian basic random variables whose distribution is closer to that of the random variables being expanded, thus allowing good approximations of lower order. As a consequence, such expansions involve polynomials orthogonal with respect to non-Gaussian measures in place of the Hermite polynomials. In principle, a sequence of orthonormal polynomials exists for any probability distribution on $\mathbb{R}$ possessing finite moments of all orders. In a series of papers [47-51] Karniadakis 
and Xiu proposed using polynomials from the Askey scheme of hypergeometric orthogonal polynomials, for which they introduced the term generalized polynomial chaos expansion. In the following, we restrict ourselves to continuous (i.e., non-discrete) distributions, which suffices for most applications and avoids certain technical difficulties.

We thus consider chaos expansions with respect to a countable sequence $\left\{\xi_{m}\right\}_{m \in \mathbb{N}}$ of (not necessarily identically distributed) basic random variables which satisfy the following assumptions:

\section{Assumption 3.1.}

(i) Each basic random variable $\xi_{m}$ possesses finite moments of all orders, i.e., $\left\langle\left|\xi_{m}\right|^{k}\right\rangle\langle\infty$ for all $k, m \in \mathbb{N}$.

(ii) The distribution functions $F_{\xi_{m}}(x):=P\left(\xi_{m} \leq x\right)$ of the basic random variables are continuous.

The linear subspaces of $\mathrm{L}^{2}(\Omega, \mathfrak{A}, P)$ spanned by polynomials of arbitrary order in such families of basic random variables are always infinite dimensional. Furthermore, any random variable which can be represented by a (multivariate) polynomial in the basic random variables either possesses both properties in Assumption 3.1 or reduces to a constant.

\subsection{One basic random variable}

As a first step, we consider expansions in a single basic random variable $\xi$ with distribution function $F_{\xi}$ satisfying Assumption 3.1. For any random variable $\eta \in \mathrm{L}^{2}(\Omega, \sigma(\xi), P)$ which is measurable with respect to $\xi$ there exists, by the Doob-Dynkin Lemma (see e.g. [19], Lem. 1.13), a measurable function $f: \mathbb{R} \rightarrow \mathbb{R}$ such that $\eta=f(\xi)$.

The distribution of the random variable $\xi$ defines a measure on the real line resulting in the probability space $\left(\mathbb{R}, \mathfrak{B}(\mathbb{R}), F_{\xi}(\mathrm{d} x)\right)$ on the range of $\xi$, where $\mathfrak{B}(\mathbb{R})$ denotes the Borel $\sigma$-algebra on $\mathbb{R}$. Since all moments of this measure are finite by assumption, this defines a sequence of orthonormal polynomials $\left\{p_{n}\right\}_{n \in \mathbb{N}_{0}}$ associated with this measure, which can be made unique, e.g., by requiring that the leading coefficient be positive. These polynomials may be generated by orthonormalizing the monomials via the Gram-Schmidt procedure or directly by the usually more stable Stieltjes process.

The sequence of random variables $\left\{p_{n}(\xi)\right\}_{n \in \mathbb{N}_{0}}$ then constitutes an orthonormal system in the Hilbert space $\mathrm{L}^{2}(\Omega, \sigma(\xi), P)$, as does the sequence $\left\{p_{n}\right\}_{n \in \mathbb{N}_{0}}$ in the Hilbert space $\mathrm{L}^{2}\left(\mathbb{R}, \mathfrak{B}(\mathbb{R}), F_{\xi}(\mathrm{d} x)\right)$, and the question of approximability by generalized polynomial chaos expansions in a single random variable $\xi$ is equivalent with the completeness of these two sequences, i.e., whether they lie dense in their respective Hilbert spaces.

The completeness of these systems is characterized by a classical theorem due to Riesz [33], which reduces the question of density of polynomials in an $\mathrm{L}^{2}$-space to the unique solvability of a moment problem.

Definition 3.2. One says that the moment problem is uniquely solvable for a probability distribution on $(\mathbb{R}, \mathfrak{B}(\mathbb{R})$ ), or that the distribution is determinate (in the Hamburger sense), if the distribution function is uniquely defined by the sequence of its moments

$$
\mu_{k}:=\left\langle\xi^{k}\right\rangle=\int_{\mathbb{R}} x^{k} F_{\xi}(\mathrm{d} x), \quad k \in \mathbb{N}_{0} .
$$

In other words, if the moment problem is uniquely solvable then no other probability distribution can have the same moment sequence. Riesz showed in [33] that the polynomials are dense in $\mathrm{L}_{\alpha}^{2}(\mathbb{R})$ for a positive Radon measure $\alpha$ if and only if the measure $\mathrm{d} \alpha(x) /\left(1+x^{2}\right)$ is determinate. For random variables $\xi$ with continuous distribution function $F_{\xi}$ (cf. Assumption 3.1) it can be shown that the polynomials are dense in $\mathrm{L}^{2}(\Omega, \sigma(\xi), P)$, and thus also in $\mathrm{L}^{2}\left(\mathbb{R}, \mathfrak{B}(\mathbb{R}), F_{\xi}(\mathrm{d} x)\right)$, if and only if $F_{\xi}$ is determinate. A proof of this equivalence can be found, e.g., in the monograph of Freud [12], Theorem 4.3, Section II.4. Additional results and background material on the moment problem and polynomial density can be found in $[4,5]$ as well as the references included therein. We summarize these facts in the following theorem. 
Theorem 3.3. The sequence of orthogonal polynomials associated with a real random variable $\xi$ satisfying Assumption 3.1 is dense in $\mathrm{L}^{2}\left(\mathbb{R}, \mathfrak{B}(\mathbb{R}), F_{\xi}(\mathrm{d} x)\right)$ if and only if the moment problem is uniquely solvable for its distribution.

Thus, if this condition is satisfied, then the sequence of random variables $\left\{p_{n}(\xi)\right\}_{n \in \mathbb{N}_{0}}$ constitutes an orthonormal basis of the Hilbert space $\mathrm{L}^{2}(\Omega, \sigma(\xi), P)$ and each element (i.e., each random variable or, more precisely, each equivalence class of random variables) of this space can be expanded with respect to this basis in an abstract Fourier series

$$
\eta=f(\xi)=\lim _{n \rightarrow \infty} \sum_{k=0}^{n} a_{k} p_{k}(\xi)=\sum_{k=0}^{\infty} a_{k} p_{k}(\xi),
$$

where the limit is in quadratic mean and the coefficients can be calculated as

$$
a_{k}=\left\langle\eta p_{k}(\xi)\right\rangle=\left\langle f(\xi) p_{n}(\xi)\right\rangle=\int_{\mathbb{R}} f(x) p_{n}(x) F_{\xi}(\mathrm{d} x), \quad k \in \mathbb{N}_{0} .
$$

The additional properties of Wiener-Hermite expansions listed in Theorem 2.2 remain valid also in this setting.

The following theorem collects several known sufficient conditions ensuring the unique solvability of the moment problem in the Hamburger sense (see, e.g., [12], Sect. II.5., [15,24,40]). Basic properties of the moment generating function can be found, e.g., in [9].

Theorem 3.4. If one of the following conditions for the distribution $F_{\xi}$ of a random variable $\xi$ satisfying Assumption 3.1 is valid, then the moment problem is uniquely solvable and therefore the set of polynomials in the random variable $\xi$ is dense in the space $\mathrm{L}^{2}(\Omega, \sigma(\xi), P)$.

(a) The distribution $F_{\xi}$ has compact support, i.e., there exists a compact interval $[a, b], a, b \in \mathbb{R}$, such that $P(\xi \in[a, b])=1$.

(b) The moment sequence $\left\{\mu_{n}\right\}_{n \in \mathbb{N}_{0}}$ of the distribution satisfies

$$
\liminf _{n \rightarrow \infty} \frac{\sqrt[2 n]{\mu_{2 n}}}{2 n}<\infty
$$

(c) The random variable is exponentially integrable, i.e., there holds

$$
\langle\exp (a|\xi|)\rangle=\int_{\mathbb{R}} \exp (a|x|) F_{\xi}(\mathrm{d} x)<\infty
$$

for a strictly positive number a. An equivalent condition is the existence of a finite moment-generating function in a neighbourhood of the origin.

(d) (Carleman's condition) The moment sequence $\left\{\mu_{n}\right\}_{n \in \mathbb{N}_{0}}$ of the distribution satisfies

$$
\sum_{n=0}^{\infty} \frac{1}{\sqrt[2 n]{\mu_{2 n}}}=\infty
$$

(e) (Lin's condition) If the distribution has a symmetric, differentiable and strictly positive density $f_{\xi}$ and for a real number $x_{0}>0$ there holds

$$
\int_{-\infty}^{\infty} \frac{-\log f_{\xi}(x)}{1+x^{2}} \mathrm{~d} x=\infty \quad \text { and } \quad \frac{-x f_{\xi}^{\prime}(x)}{f_{\xi}(x)} \nearrow \infty\left(x \rightarrow \infty, x \geq x_{0}\right) .
$$


If, in Lin's condition, the integral for a probability distribution with strictly positive density is finite, then the distribution is indeterminate (Krein's condition).

Examples of probability distributions, for which the moment problem is uniquely solvable are the uniform, beta, gamma and the normal distributions. By contrast, the moment problem is not uniquely solvable for the lognormal distribution, so that the sequence of random variables $\left\{p_{n}(\xi)\right\}_{n \in \mathbb{N}_{0}}$ for a lognormal random variable $\xi$ does not constitute a basis of the Hilbert space $\mathrm{L}^{2}(\Omega, \sigma(\xi), P)$, and hence there will be some elements (random variables) in this space which are not the limit of their generalized polynomial chaos expansion.

Further examples of random variables with indeterminate distribution are certain powers of random variables with normal or gamma distribution (see, e.g., $[36,40])$. Note that the expansion (3.1) still converges in quadratic mean, but its limit may be a second-order random variable different from $\eta$. In this case the convergence of the generalized polynomial chaos expansions to the desired limit must be shown in a different way.

Remark 3.5. For random variables with discrete or mixed distributions, which are excluded by Assumption 3.1, we note that results given in [12], Theorem 4.3 as well as in the remark following Theorem 2.2 of the same work, show that the determinacy of discrete or mixed distributions is also sufficient for the density of polynomials in $L^{2}$ in that case.

\subsection{Finitely many basic random variables}

We now turn to the case in which the stochasticity of the underlying problem is characterized by a finite number of independent random variables $\xi_{1}, \xi_{2}, \ldots, \xi_{M}$, which we collect in the random vector $\boldsymbol{\xi}: \Omega \rightarrow \mathbb{R}^{M}$. This situation is often referred to as finite-dimensional noise in the stochastic finite element literature, and typically arises when a random field is approximated by a truncated Karhunen-Loï $i \frac{1}{2}$ ve expansion. Denoting by $\left\{p_{j}^{(m)}\right\}_{j \in \mathbb{N}_{0}}, m=1, \ldots, M$, the sequence of polynomials orthonormal with respect to the distribution of $\xi_{m}$, we note that the set of multivariate (tensor product) polynomials given by

$$
p_{\boldsymbol{\alpha}}(\boldsymbol{\xi})=\prod_{m=1}^{M} p_{\alpha_{m}}^{(m)}\left(\xi_{m}\right), \quad \boldsymbol{\alpha}=\left(\alpha_{1}, \ldots, \alpha_{M}\right) \in \mathbb{N}_{0}^{M},
$$

constitutes an orthonormal system of random variables in the space $\mathrm{L}^{2}(\Omega, \sigma(\boldsymbol{\xi}), P)$. By consequence, the polynomials

$$
p_{\boldsymbol{\alpha}}: \mathbf{x} \mapsto p_{\boldsymbol{\alpha}}(\mathbf{x}), \quad \boldsymbol{\alpha} \in \mathbb{N}_{0}^{M},
$$

form an orthonormal system in the image space $\mathrm{L}^{2}\left(\mathbb{R}^{M}, \mathfrak{B}\left(\mathbb{R}^{M}\right)\right)$ endowed with the product probability measure $F_{\xi_{1}}\left(\mathrm{~d} x_{1}\right) \times \cdots \times F_{\xi_{M}}\left(\mathrm{~d} x_{M}\right)$. As is well known, tensor products of systems of orthonormal bases of separable Hilbert spaces form an orthonormal basis of the tensor product Hilbert space (see e.g. [32], Sect. II.4, or [31]), which implies the following result:

Theorem 3.6. Let $\boldsymbol{\xi}=\left(\xi_{1}, \ldots, \xi_{M}\right)$ be a vector of $M \in \mathbb{N}$ independent random variables satisfying Assumption 3.1 and $\left\{p_{j}^{(m)}\right\}_{j \in \mathbb{N}_{0}}, m=1, \ldots, M$, the associated orthonormal polynomial sequences. Then the orthonormal system of random variables

$$
p_{\boldsymbol{\alpha}}(\boldsymbol{\xi})=\prod_{m=1}^{M} p_{\alpha_{m}}^{(m)}\left(\xi_{m}\right), \quad \boldsymbol{\alpha} \in \mathbb{N}_{0}^{M},
$$

is an orthonormal basis of the space $\mathrm{L}^{2}(\Omega, \sigma(\boldsymbol{\xi}), P)$ if and only if the moment problem is uniquely solvable for each random variable $\xi_{m}, m=1, \ldots, M$. In this case any random variable $\eta \in \mathrm{L}^{2}(\Omega, \sigma(\boldsymbol{\xi}), P)$ can be expanded in an abstract Fourier series of multivariate orthonormal polynomials in the basic random variables, the generalized polynomial chaos expansion

$$
\eta=\sum_{\boldsymbol{\alpha} \in \mathbb{N}_{0}^{M}} a_{\boldsymbol{\alpha}} p_{\boldsymbol{\alpha}}(\boldsymbol{\xi}) \quad \text { with coefficients } a_{\boldsymbol{\alpha}}=\left\langle\eta p_{\boldsymbol{\alpha}}(\boldsymbol{\xi})\right\rangle
$$


In other words, the set of multivariate tensor product polynomials (3.3) in a finite number of independent random variables $\xi_{1}, \ldots, \xi_{M}$ is dense in $\mathrm{L}^{2}(\Omega, \sigma(\boldsymbol{\xi}), P)$, as are the $M$-variate polynomials in the space $\mathrm{L}^{2}\left(\mathbb{R}^{M}, \mathfrak{B}\left(\mathbb{R}^{M}\right), F_{\xi_{1}}\left(\mathrm{~d} x_{1}\right) \times \cdots \times F_{\xi_{M}}\left(\mathrm{~d} x_{M}\right)\right)$, if and only if each sequence $\left\{p_{j}^{(m)}(\xi)\right\}_{j \in \mathbb{N}_{0}}$ is dense in $\mathrm{L}^{2}\left(\Omega, \sigma\left(\xi_{m}\right), P\right)$ for $m=1,2, \ldots, M$.

If the basic random variables are not independent, then the construction of a sequence of orthonormal polynomials is still always possible. In this case, however, the tensor product structure of the polynomial space is lost and additional difficulties arise. In particular, the sequence of orthonormal polynomials is no longer uniquely defined, but depends on the ordering of the monomials. Furthermore, the link between the determinacy of the distribution and the density of polynomials in the associated $\mathrm{L}^{2}$ spaces becomes more intricate, and conditions on the determinacy of such distributions are more intricate (for more about these and related issues, see, e.g., $[4,31,34,53])$. We therefore restrict ourselves here to simple sufficient conditions for the density of multivariate polynomials in the corresponding $\mathrm{L}^{2}$ spaces. These will generally suffice in practical applications.

Theorem 3.7. If the distribution function $F_{\boldsymbol{\xi}}$ of a random vector $\boldsymbol{\xi}=\left(\xi_{1}, \ldots, \xi_{M}\right)$ with continuous distribution and finite moments of all orders satisfies one of the following conditions, then the multivariate polynomials in $\xi_{1}, \ldots, \xi_{M}$ are dense in $\mathrm{L}^{2}(\Omega, \sigma(\boldsymbol{\xi}), P)$. In this case any random variable $\eta \in \mathrm{L}^{2}(\Omega, \sigma(\boldsymbol{\xi}), P)$ is the limit of its generalized polynomial chaos expansion, which converges in quadratic mean.

(a) The distribution function $F_{\boldsymbol{\xi}}$ has compact support, i.e., there exists a compact set $K \subset \mathbb{R}^{M}$ such that $P(\boldsymbol{\xi} \in K)=1$.

(b) The random vector is exponentially integrable, i.e., there exists a $>0$ such that

$$
\langle\exp (a\|\boldsymbol{\xi}\|)\rangle=\int_{\mathbb{R}^{M}} \exp (a\|\mathbf{x}\|) F_{\boldsymbol{\xi}}(\mathrm{d} \mathbf{x})<\infty
$$

where $\|\cdot\|$ denotes any norm on $\mathbb{R}^{M}$.

Proof. By a result of Petersen (see [31], Thm. 3) the distribution of the random vector $\boldsymbol{\xi}=\left(\xi_{1}, \ldots, \xi_{M}\right)$ is determinate if the distribution of each random variable $\xi_{m}, m=1, \ldots, M$, is determinate. Moreover, the set of multivariate polynomials is dense in $L^{q}\left(\mathbb{R}^{M}, \mathfrak{B}\left(\mathbb{R}^{M}\right), F_{\boldsymbol{\xi}}(\mathrm{d} x)\right)$ for any $1 \leq q<p$ if the polynomials are dense in $L^{p}\left(\mathbb{R}, \mathfrak{B}(\mathbb{R}), F_{\xi_{m}}\left(\mathrm{~d} x_{m}\right)\right)$ for each $m=1, \ldots, M$ (the proposition following Thm. 3 in [31]). But if the exponential integrability condition is satisfied, then it is satisfied for each random variable $\xi_{m}, m=1, \ldots, M$. Now by Theorem 6 in [5], the polynomials are dense in the space $L^{p}\left(\mathbb{R}, \mathfrak{B}(\mathbb{R}), F_{\xi_{m}}\left(\mathrm{~d} x_{m}\right)\right)$ for each $p \geq 1$.

\subsection{Infinitely many basic random variables}

We now consider the situation where the stochasticity of the underlying problem is characterized by a countable sequence $\left\{\xi_{m}\right\}_{m \in \mathbb{N}}$ of random variables of which each satisfies Assumption 3.1, all defined on a fixed, sufficiently rich probability space $(\Omega, \mathfrak{A}, P)$.

As in the case of Gaussian polynomial chaos, we define the following subspaces of $\operatorname{L}^{2}(\Omega, \mathfrak{A}, P)$ for $M \in \mathbb{N}$ and $n \in \mathbb{N}_{0}$ :

$$
\begin{aligned}
& \mathscr{P}_{n}^{M}:=\left\{p\left(\xi_{1}, \ldots, \xi_{M}\right): p \text { a polynomial of degree } \leq n\right\}, \\
& \widetilde{\mathscr{P}}^{M}:=\bigcup_{n=0}^{\infty} \mathscr{P}_{n}^{M}, \quad \mathscr{P}_{n}:=\bigcup_{M=1}^{\infty} \mathscr{P}_{n}^{M}, \quad \widetilde{\mathscr{P}}:=\bigcup_{n=0}^{\infty} \mathscr{P}_{n} .
\end{aligned}
$$

Furthermore we denote the relevant $\sigma$-algebras

$$
\mathfrak{A}_{M}:=\sigma\left(\left\{\xi_{m}\right\}_{m=1}^{M}\right), M \in \mathbb{N}, \quad \text { and } \quad \mathfrak{A}_{\infty}:=\sigma\left(\left\{\xi_{m}\right\}_{m \in \mathbb{N}}\right) .
$$


We then have the inclusions

$$
\begin{aligned}
\mathscr{P}_{n}^{M} \subset \widetilde{\mathscr{P}}^{M} \subset \mathrm{L}^{2}\left(\Omega, \mathfrak{A}_{M}, P\right), & & n \in \mathbb{N}_{0}, M \in \mathbb{N}, \\
\mathscr{P}_{n} \subset \overline{\mathscr{P}_{n}} \subset \widetilde{\mathscr{P}} \subset \mathrm{L}^{2}\left(\Omega, \mathfrak{A}_{\infty}, P\right), & & n \in \mathbb{N}_{0} .
\end{aligned}
$$

For $M \in \mathbb{N}$ the set $\widetilde{\mathscr{P}}^{M}$ is the closed linear subspace of $\mathrm{L}^{2}\left(\Omega, \mathfrak{A}_{M}, P\right)$ containing all $\mathrm{L}^{2}$-limits of polynomials in the basic random variables $\left(\xi_{1}, \ldots, \xi_{M}\right)$, and the set $\widetilde{P}$ is the closed linear subspace of $\mathrm{L}^{2}\left(\Omega, \mathfrak{A}_{\infty}, P\right)$ containing all $\mathrm{L}^{2}$-limits of polynomials in all basic random variables $\left\{\xi_{m}\right\}_{m \in \mathbb{N}}$. Theorem 3.8 below asserts that a sufficient condition for the polynomials in all basic random variables $\left\{\xi_{m}\right\}_{m \in \mathbb{N}}$ to be dense in $\mathrm{L}^{2}\left(\Omega, \mathfrak{A}_{\infty}, P\right)$ is that the polynomials in each finite subset $\left\{\xi_{m}\right\}_{m=1}^{M}$ of the basic random variables be dense in $\operatorname{L}^{2}\left(\Omega, \mathfrak{A}_{M}, P\right)$.

Theorem 3.8. If

$$
\widetilde{\mathscr{P}}^{M}=\mathrm{L}^{2}\left(\Omega, \mathfrak{A}_{M}, P\right) \quad \text { for all } M \in \mathbb{N},
$$

then $\widetilde{P}=\mathrm{L}^{2}\left(\Omega, \mathfrak{A}_{\infty}, P\right)$.

Proof. We show that under the assumption (3.4) any random variable $\eta$ in the orthogonal complement of $\widetilde{\mathscr{P}}$ in $\mathrm{L}^{2}\left(\Omega, \mathfrak{A}_{\infty}, P\right)$ must vanish. Otherwise any such random variable $\eta$ can be normalized such that $\left\langle\eta^{2}\right\rangle=1$. The union $\cup_{M=1}^{\infty} \mathrm{L}^{2}\left(\Omega, \mathfrak{A}_{M}, P\right)$ of the nested sequence of $\mathrm{L}^{2}$-spaces lies dense in $\mathrm{L}^{2}\left(\Omega, \mathfrak{A}_{\infty}, P\right)$ (see e.g. [6], p. 109, Cor. 3.6.8). Therefore, given $\epsilon>0$, there exists $\eta_{0} \in \mathrm{L}^{2}\left(\Omega, \mathfrak{A}_{M_{0}}, P\right)$ with $M_{0}$ sufficiently large such that

$$
\left\|\eta-\eta_{0}\right\|_{\mathrm{L}^{2}}<\epsilon
$$

By the reverse triangle inequality this implies

$$
\left\|\eta_{0}\right\|_{\mathrm{L}^{2}} \geq\|\eta\|_{\mathrm{L}^{2}}-\left\|\eta-\eta_{0}\right\|_{\mathrm{L}^{2}} \geq 1-\epsilon .
$$

On the other hand, since $\eta_{0} \in \mathrm{L}^{2}\left(\Omega, \mathfrak{A}_{M_{0}}, P\right)=\widetilde{\mathscr{P}} M_{0} \subset \widetilde{\mathscr{P}} \perp \eta$, we also have

$$
\left\|\eta-\eta_{0}\right\|_{\mathrm{L}^{2}}^{2}=\|\eta\|_{\mathrm{L}^{2}}^{2}+\left\|\eta_{0}\right\|_{\mathrm{L}^{2}}^{2} \geq 1+(1-\epsilon)^{2},
$$

which contradicts (3.5) for sufficiently small $\epsilon$.

Corollary 3.9. Let $\left\{\xi_{m}\right\}_{m \in \mathbb{N}}$ be a sequence of basic random variables satisfying Assumption 3.1 and $\eta \in$ $\mathrm{L}^{2}\left(\Omega, \mathfrak{A}_{\infty}, P\right)$. If for each $M \in \mathbb{N}$ the polynomials in $\left\{\xi_{m}\right\}_{m=1}^{M}$ are dense in $\mathrm{L}^{2}\left(\Omega, \mathfrak{A}_{M}, P\right)$, then the generalized polynomial chaos expansion of $\eta$ converges to $\eta$ in quadratic mean.

Polynomial chaos expansions and generalized polynomial chaos expansions generally work with basic random variables which are, in addition, independent. In this case the sufficient condition given in Theorem 3.6 is also necessary. Moreover, the density result is then equivalent to the density of each univariate family of polynomials.

Corollary 3.10. Let $\left\{\xi_{m}\right\}_{m \in \mathbb{N}}$ be a sequence of independent basic random variables satisfying Assumption 3.1 and $\eta \in \mathrm{L}^{2}\left(\Omega, \mathfrak{A}_{\infty}, P\right)$. Then the generalized polynomial chaos expansion of $\eta$ converges in quadratic mean to the random variable $\eta$ if and only if the moment problem for the distribution of each random variable $\xi_{m}$ is uniquely solvable (or, equivalently, the polynomials in the random variable $\xi_{m}$ are dense in $\mathrm{L}^{2}\left(\Omega, \sigma\left(\xi_{m}\right), P\right)$ for each $m \in \mathbb{N}$ ).

Proof. If for each $m \in \mathbb{N}$ the moment problem for the distribution of the random variable $\xi_{m}$ is uniquely solvable and, equivalently the set of polynomials in the random variable $\xi_{m}$ is dense in $\mathrm{L}^{2}\left(\Omega, \sigma\left(\xi_{m}\right), P\right)$, then this holds by Theorem 3.6 for any finite subfamily and hence, from Theorem 3.8 the conclusion follows. In order to prove the converse statement we assume that for an index $m_{0} \in \mathbb{N}$ the polynomials in the random variable $\xi_{m_{0}}$ are not dense in $\mathrm{L}^{2}\left(\Omega, \sigma\left(\xi_{m_{0}}\right), P\right)$. Then there exists a second-order random variable $\eta_{0} \in \mathrm{L}^{2}\left(\Omega, \sigma\left(\xi_{m_{0}}\right), P\right)$ with norm 1, which cannot be approximated by polynomials in $\xi_{m_{0}}$. Due to the independence of the basic random variables, we have that polynomials in the remaining basic random variables, and therefore also their closure, are orthogonal to $\mathrm{L}^{2}\left(\Omega, \sigma\left(\xi_{m_{0}}\right), P\right)$. Consequently, such polynomials have a distance to $\eta_{0}$ of at least one. We therefore conclude that $\eta_{0} \in \mathrm{L}^{2}\left(\Omega, \mathfrak{A}_{\infty}, P\right) \backslash \widetilde{\mathscr{P}}$. 
Remark 3.11. If the basic random variables $\left\{\xi_{m}\right\}_{m \in \mathbb{N}}$ are not independent, it may happen that for a finite number $M_{0} \in \mathbb{N}$, we have $\widetilde{\mathscr{P}} M_{0} \subsetneq \mathrm{L}^{2}\left(\Omega, \mathfrak{A}_{M_{0}}, P\right)$ but $\widetilde{P}=\mathrm{L}^{2}\left(\Omega, \mathfrak{A}_{\infty}, P\right)$. As an example, take an infinite sequence of independent and normalized basic variables $\left\{\xi_{m}\right\}_{m \in \mathbb{N}}$ satisfying Assumption 3.1, such that the distribution of $\xi_{1}$ is indeterminate while those of the remaining random variables are determinate. Furthermore choose a sequence $\left\{\zeta_{m}\right\}_{m \in \mathbb{N}}$ of random variables such that the set $\left\{\xi_{1}, \zeta_{j} ; j \in \mathbb{N}\right\}$ is an orthonormal basis of the Hilbert space $\mathrm{L}^{2}\left(\Omega, \sigma\left(\xi_{1}\right), P\right)$. This is possible because this space is separable. Then arrange a countable number of random variables, e.g. by the rule $\widetilde{\xi}_{2 k-1}:=\xi_{k}, \widetilde{\xi}_{2 k}:=\zeta_{k}, k \in \mathbb{N}$ and consider this sequence $\left\{\widetilde{\xi}_{i}\right\}_{i \in \mathbb{N}}$ as a sequence of basic random variables. Then we have $\widetilde{\mathscr{P}}^{1} \neq \mathrm{L}^{2}\left(\Omega, \mathfrak{A}_{1}, P\right)$ but

$$
\widetilde{\mathscr{P}}=\mathrm{L}^{2}\left(\Omega, \mathfrak{A}_{\infty}, P\right)=\bigoplus_{m=1}^{\infty} \mathrm{L}^{2}\left(\Omega, \sigma\left(\xi_{m}\right), P\right) .
$$

Remark 3.12. We note that all preceding results, although phrased in terms of real-valued random variables in $L^{2}(\Omega, \mathfrak{A}, P)$, extend without difficulty to the expansion of random variables taking values in a separable Hilbert space $\left(X,(\cdot, \cdot)_{X}\right)$ with orthonormal basis $\left\{x_{n}\right\}_{n \in \mathbb{N}}$. This can be seen as follows: an $X$-valued random variable $\eta=\sum_{n=1}^{\infty} \eta_{n} x_{n}$, for which $\left\langle\|\eta\|_{X}^{2}\right\rangle<\infty$, possesses coefficients $\eta_{n}=\left(\eta, x_{n}\right)_{X}$ which are in $L^{2}(\Omega, \mathfrak{A}, P)$ since $\left|\eta_{n}\right| \leq\|\eta\|_{X}$ by the Cauchy-Schwarz inequality and therefore, taking expectations,

$$
\left\langle\left|\eta_{n}\right|^{2}\right\rangle \leq\left\langle\|\eta\|_{X}^{2}\right\rangle<\infty
$$

Each coefficient $\eta_{n}$ thus has a convergent generalized polynomial chaos expansion in terms of any sequence of basic random variables $\left\{\xi_{m}\right\}_{m \in \mathbb{N}}$ with determinate distributions, i.e.,

$$
\eta_{n}=\sum_{\alpha \in \mathscr{I}} a_{n, \boldsymbol{\alpha}} p_{\boldsymbol{\alpha}}(\boldsymbol{\xi}), \quad a_{n, \boldsymbol{\alpha}}:=\left\langle\eta_{n} p_{\boldsymbol{\alpha}}(\boldsymbol{\xi})\right\rangle, \quad \mathscr{I}=\left\{\boldsymbol{\alpha} \in \mathbb{N}_{0}^{\mathbb{N}}:|\boldsymbol{\alpha}|:=\alpha_{1}+\alpha_{2}+\cdots<\infty\right\},
$$

with respect to a sequence of multivariate orthonormal polynomials ${ }^{6}\left\{p_{\boldsymbol{\alpha}}\right\}_{\boldsymbol{\alpha} \in \mathscr{I}}$ in the basic random variables $\left\{\xi_{m}\right\}_{m \in \mathbb{N}}$. Combining these expansions yields the convergent generalized polynomial chaos expansion of $\eta$ as

$$
\eta=\sum_{n=1}^{\infty}\left(\sum_{\alpha \in \mathscr{I}} a_{n, \boldsymbol{\alpha}} p_{\boldsymbol{\alpha}}(\boldsymbol{\xi})\right) x_{n}=\sum_{\alpha \in \mathscr{I}}\left(\sum_{n=1}^{\infty} a_{n, \boldsymbol{\alpha}} x_{n}\right) p_{\boldsymbol{\alpha}}(\boldsymbol{\xi}),
$$

where the order of summation may be interchanged since both series derive from expansions in orthonormal bases. We note that the limits represented by the outer sums in (3.6) are both in the sense of mean-square convergence in $X$, i.e., that $\left\langle\left\|\eta-\sum_{n=1}^{N} \eta_{n} x_{n}\right\|_{X}^{2}\right\rangle \rightarrow 0$ as $N \rightarrow \infty$ as well as $\left\langle\left\|\eta-\sum_{|\boldsymbol{\alpha}| \leq K}\left\langle\eta p_{\boldsymbol{\alpha}}(\boldsymbol{\xi})\right\rangle p_{\boldsymbol{\alpha}}(\boldsymbol{\xi})\right\|_{X}^{2}\right\rangle \rightarrow 0$ as $K \rightarrow \infty$. Moreover, in complete analogy to the scalar case, the term in parentheses following the second equality in (3.6) is the expansion coefficient $\left\langle\eta p_{\boldsymbol{\alpha}}(\boldsymbol{\xi})\right\rangle \in X$ of $\eta$ with multi-index $\boldsymbol{\alpha}$.

\section{EXAMPLES}

In this section we present a number of examples intended to illustrate the preceding results and indicate how they are relevant for stochastic Galerkin approximations, for which generalized polynomial chaos expansions were originally developed.

\footnotetext{
${ }^{6}$ These can be constructed in the same way as in the case of finitely many basic random variables, since they involve only finite products of univariate orthonormal polynomials.
} 


\subsection{Periodic functions of a lognormal random variable}

As noted in Section 3.1, the lognormal distribution is not determinate, i.e., its moment problem fails to possess a unique solution. By consequence, polynomials in a lognormal random variable $\eta$ are not dense in $\mathrm{L}^{2}(\Omega, \sigma(\eta), P)$. We give an example of a nontrivial class of functions in the orthogonal complement of the span of these polynomials.

Denote by $\xi \sim N(0,1)$ a standard Gaussian random variable and recall that the density function of the lognormal random variable $\eta:=\mathrm{e}^{\xi}$ is given by

$$
f_{\eta}(x)= \begin{cases}\frac{1}{x \sqrt{2 \pi}} \mathrm{e}^{-\frac{\log ^{2} x}{2}}, & x>0 \\ 0, & \text { otherwise. }\end{cases}
$$

Proposition 4.1. Let $\eta$ be a lognormal random variable with density (4.1). Then for any function $g: \mathbb{R} \rightarrow \mathbb{R}$ which is measurable, odd and 1-periodic, i.e., $g(y+1)=g(y)$ and for which $\left\langle g(\log (\eta))^{2}\right\rangle<\infty$, there holds

$$
\left\langle\eta^{k} g(\log \eta)\right\rangle=\int_{0}^{\infty} x^{k} f_{\eta}(x) g(\log x) \mathrm{d} x=0 \quad \forall k \in \mathbb{N}_{0} .
$$

Proof. The change of variables $y=\log x$ yields, for all $k \in \mathbb{N}_{0}$,

$$
\begin{aligned}
\int_{0}^{\infty} x^{k} \frac{1}{x \sqrt{2 \pi}} \mathrm{e}^{-\frac{\log ^{2} x}{2}} g(\log x) \mathrm{d} x & =\frac{1}{\sqrt{2 \pi}} \int_{-\infty}^{\infty} \mathrm{e}^{k y} \mathrm{e}^{-\frac{y^{2}}{2}} g(y) \mathrm{d} y \\
& =\frac{\mathrm{e}^{\frac{k^{2}}{2}}}{\sqrt{2 \pi}} \int_{-\infty}^{\infty} \mathrm{e}^{-\frac{(y-k)^{2}}{2}} g(y) \mathrm{d} y=\frac{\mathrm{e}^{\frac{k^{2}}{2}}}{\sqrt{2 \pi}} \int_{-\infty}^{\infty} \mathrm{e}^{-\frac{z^{2}}{2}} g(z+k) \mathrm{d} z \\
& =\frac{\mathrm{e}^{\frac{k^{2}}{2}}}{\sqrt{2 \pi}} \int_{-\infty}^{\infty} \mathrm{e}^{-\frac{z^{2}}{2}} g(z) \mathrm{d} z=0,
\end{aligned}
$$

where we have substituted $z=y-k$ in the third identity and subsequently used the periodicity and the oddness of $g$.

Note that the set of all random variables of the form $g(\log \eta)$ with $g$ as in Proposition 4.1 constitutes a (nontrivial) linear subspace of $\mathrm{L}^{2}(\Omega, \sigma(\eta), P)$, and that $(4.2)$ extends to the closure of this subspace. An immediate consequence of (4.2) is that the generalized polynomial chaos coefficients of the random variable $g(\log \eta)$ with respect to the lognormal random variable $\eta$ must also all vanish. The limit of this expansion is therefore zero, which does not coincide with the random variable under expansion.

Specifically, the nonzero function $g(x)=\sin (2 \pi x)$, a popular example for non-determinacy $c f$. [36,40], satisfies the requirements of Proposition 4.1. The generalized polynomial chaos expansion of $g(\log \eta)$ with respect to the lognormal random variable $\eta$ therefore fails to converge in quadratic mean to the random variable $g(\log \eta)$. By contrast, the (classical) polynomial chaos expansion of $g(\log \eta)$ with respect to the Gaussian random variable $\xi=\log \eta$ is mean-square convergent to $g(\log (\eta))=g(\xi)$. This expansion is given by

$$
\sin (2 \pi \log \eta)=\sum_{k=0}^{\infty} a_{k} h_{k}(\log \eta), \quad \text { where } a_{k}= \begin{cases}\frac{(-1)^{(k-1) / 2}(2 \pi)^{k}}{\sqrt{k !}} \mathrm{e}^{-2 \pi^{2}}, & k \text { odd }, \\ 0, & k \text { even }\end{cases}
$$

and $\left\{h_{k}\right\}_{k \in \mathbb{N}_{0}}$ denote the normalized ("probabilist's") Hermite polynomials given by their Rodrigues' formula

$$
h_{k}(x)=\frac{1}{\sqrt{k !}} H_{k}(x), \quad H_{k}(x)=(-1)^{k} \mathrm{e}^{\frac{x^{2}}{2}}\left(\frac{\mathrm{d}^{k}}{\mathrm{~d} x^{k}} \mathrm{e}^{-\frac{x^{2}}{2}}\right), \quad x \in \mathbb{R},
$$

which are orthonormal with respect to the standard Gaussian density function

$$
f_{\xi}(x)=\frac{1}{\sqrt{2 \pi}} \mathrm{e}^{-\frac{x^{2}}{2}} .
$$




\subsection{The reciprocal of a lognormal random variable}

Before proceeding with the next example we give an explicit representation of the orthonormal polynomials associated with the lognormal density (4.1). These can be constructed in terms of Stieltjes-Wigert polynomials (cf. [41], Sect. 2.7), which are orthogonal with respect to the family of weight functions

$$
w_{\nu}(x)=\frac{\nu}{\sqrt{\pi}} \mathrm{e}^{-\nu^{2} \log ^{2} x}, \quad x>0, \quad \nu>0 .
$$

For the details of this construction we refer to Appendix A. The coefficients $\alpha_{k}$ and $\beta_{k}$ of the associated three-term recurrence

$$
\begin{aligned}
& p_{-1}(x) \equiv 0, \quad p_{0}(x) \equiv 1 \\
& \sqrt{\beta_{k+1}} p_{k+1}(x)=\left(x-\alpha_{k}\right) p_{k}(x)-\sqrt{\beta_{k}} p_{k-1}(x), \quad k \geq 0
\end{aligned}
$$

are found to be (cf. [37])

$$
\alpha_{k}=\left(\mathrm{e}^{k}(\mathrm{e}+1)-1\right) \mathrm{e}^{(2 k-1) / 2}, \quad \beta_{k}=\left(\mathrm{e}^{k}-1\right) \mathrm{e}^{3 k-2} .
$$

Using these, the generalized polynomial chaos expansion coefficients of the random variable

$$
\zeta:=\frac{1}{\eta}
$$

are found to be

$$
a_{0}=\mathrm{e}^{1 / 2}, \quad a_{k}=(-1)^{k} \mathrm{e}^{-\left(k^{2}+3 k-2\right) / 4} \sqrt{\prod_{i=1}^{k}\left(\mathrm{e}^{i}-1\right)}, \quad k \geq 1 .
$$

A derivation of these coefficients is provided in Appendix B. We now come to a non-convergence result.

Proposition 4.2. The generalized polynomial chaos expansion of the random variable $\zeta$ defined in (4.5) with respect to the orthonormal polynomials $\left\{p_{k}\right\}_{k \in \mathbb{N}_{0}}$ in $\eta$ does not converge in mean-square to the random variable $\zeta$.

Proof. The truncated chaos expansion of order $n$

$$
\zeta_{n}:=\sum_{k=0}^{n} a_{k} p_{k}(\eta)=\mathrm{e}^{1 / 2}+\sum_{k=1}^{n}(-1)^{k} \mathrm{e}^{-\left(k^{2}+3 k-2\right) / 4} \sqrt{\prod_{i=1}^{k}\left(\mathrm{e}^{i}-1\right) p_{k}}(\eta)
$$

can be bounded as follows:

$$
\begin{aligned}
\left\|\zeta_{n}\right\|_{\mathrm{L}^{2}}^{2} & =\mathrm{e}+\sum_{k=1}^{n} \mathrm{e}^{-\left(k^{2}+3 k-2\right) / 2} \prod_{i=1}^{k}\left(\mathrm{e}^{i}-1\right) \leq \mathrm{e}+\sum_{k=1}^{n} \mathrm{e}^{-\left(k^{2}+3 k-2\right) / 2} \prod_{i=1}^{k} \mathrm{e}^{i} \\
& \leq \mathrm{e}+\sum_{k=1}^{\infty} \mathrm{e}^{-k+1}=\frac{\mathrm{e}^{2}}{\mathrm{e}-1} .
\end{aligned}
$$

By consequence, and the fact that $\|\zeta\|_{\mathrm{L}^{2}}=\mathrm{e}$, the remainder of the truncated expansion is bounded below by

$$
\left\|\zeta-\zeta_{n}\right\|_{\mathrm{L}^{2}}^{2}=\|\zeta\|_{\mathrm{L}^{2}}^{2}-\left\|\zeta_{n}\right\|_{\mathrm{L}^{2}}^{2} \geq \mathrm{e}^{2}-\frac{\mathrm{e}^{2}}{\mathrm{e}-1}>0 .
$$




\subsection{Stochastic Galerkin approximation}

We now turn to an important application of (generalized) polynomial chaos expansions, namely the approximate solution of differential equations with random data by the stochastic Galerkin method. We consider the boundary-value problem for the one-dimensional diffusion problem

$$
-\left(a u^{\prime}\right)^{\prime}=f, \quad u(0)=0, \quad\left(a u^{\prime}\right)(1)=F,
$$

posed on the unit interval $(0,1)$ where $a=a(x, \omega)$ is a given positive random field, $f=f(x)$ a deterministic function, and $F$ a given constant. The solution of (4.7) is

$$
u(x, \omega)=\int_{0}^{x} \frac{1}{a(y, \omega)}\left(F+\int_{y}^{1} f(z) \mathrm{d} z\right) \mathrm{d} y .
$$

We now compare the stochastic Galerkin procedure for solving (4.7) using a spectral element discretization in space combined with each of lognormal, Hermite and reflected Gaussian polynomial chaos expansions in the stochastic parameter, displaying non-convergence, slow convergence and fast convergence, respectively, to the solution, which we measure by the convergence of the first two moments of the solution field $u$ as a function of the spatial variable $x$.

\subsubsection{Lognormal chaos}

Consider first the case that $a$ is simply the fixed lognormal random variable $\eta(\omega)$ from the previous subsection. The solution then simplifies to

$$
u(x, \omega)=\zeta(\omega) \int_{0}^{x}\left(F+\int_{y}^{1} f(z) \mathrm{d} z\right) \mathrm{d} y
$$

i.e., it is the product of the reciprocal $\zeta$ of a lognormal random variable as defined in (4.5) with a purely deterministic function of $x$. An approximation of $u$ based on generalized polynomial chaos, i.e., expansion in the orthogonal polynomials $\left\{p_{k}\right\}$ in $\eta$, cannot converge to the solution in view of Proposition 4.2. Therefore, if the solution of the boundary value problem with random data (4.7) is approximated with a stochastic Galerkin method employing lognormal chaos in the stochastic variables, the approximation thus obtained can be no better than the best approximation provided by a truncated chaos expansion. Since the latter has been shown not to converge to the solution, the Galerkin approximation cannot do so either.

\subsubsection{Hermite and reflected Gaussian chaos}

Next, consider the same boundary value problem (4.7) with random field

$$
a(x, \omega)=\exp (|\xi(\omega)| x), \quad \xi \sim N(0,1) .
$$

The distribution of the random variable $|\xi|$, sometimes called a reflected Gaussian distribution, is determinate in the sense of Definition 3.1 by Theorem 3.3 (c). Polynomials in $|\xi|$ are therefore dense in $L^{2}(\Omega, \sigma(|\xi|), P)$ and the associated generalized polynomial chaos expansion of $u$ therefore converges to $u$ in mean square.

In the following, we compare two stochastic Galerkin approximations (see e.g. [2] for an introduction) to the solution of (4.7) based on two different types of polynomial chaos expansion: standard Hermite chaos and the generalized polynomial chaos constructed from polynomials orthogonal with respect to the reflected Gaussian distribution. In the first case we use as the trial space in the stochastic dimension the Hermite polynomials in $\xi$ up to a fixed degree $n$. In the second, we use the polynomials orthonormal with respect to the distribution of $|\xi|$ up to degree $n$. The load function $f$ in (4.7) is chosen as $f \equiv 1$ and the boundary data as $F=1$, resulting in the solution random field

$$
u(x, \xi)=\int_{0}^{x} \mathrm{e}^{-|\xi| y}(2-y) \mathrm{d} y=\mathrm{e}^{-|\xi| x}\left(\frac{1}{|\xi|^{2}}-\frac{2-x}{|\xi|}\right)-\frac{1}{|\xi|^{2}}+\frac{2}{|\xi|} .
$$


We observe that $u$ depends smoothly on $|\xi|$, but not on $\xi$ itself, indicating that expansions with respect to the variable $\eta:=|\xi|$ can be expected to converge faster than those with respect to $\xi$. The stochastic Galerkin discretization is based on the variational formulation of (4.7), which results after multiplying the differential equation with a test random field $v(x, \omega)$ and integrating by parts, and consists of seeking $u \in \mathscr{V}$ such that

$$
B(u, v)=\ell(v) \quad \forall v \in \mathscr{V},
$$

where the variational space is $\mathscr{V}:=\left\{u \in \mathrm{H}^{1}(0,1) \otimes \mathrm{L}^{2}(\Omega): u(0)=0\right.$ a.s. $\}$ and the bilinear and linear forms $B(\cdot, \cdot)$ and $\ell(\cdot)$, respectively, are given by

$$
B(u, v)=\left\langle\int_{0}^{1} a(x, \omega) u^{\prime}(x, \omega) v^{\prime}(x, \omega) \mathrm{d} x\right\rangle, \ell(v)=\left\langle\int_{0}^{1} f(x) v(x, \omega) \mathrm{d} x+v(1, \omega) F\right\rangle .
$$

We note that, due to the unboundedness of the random field $a$, the bilinear form $B(\cdot, \cdot)$ is not defined on all of $\mathscr{V} \times \mathscr{V}$. The variational problem is nonetheless well-posed, as is discussed, e.g., in [28]. The stochastic Galerkin discretization now results from restricting trial and test random fields to finite-dimensional subspaces $\mathscr{V}_{d, n}=X_{d} \otimes \Xi_{n}$ of $\mathscr{V}$ with finite-dimensional subspaces $X_{d}$ and $\Xi_{n}$ of $\mathrm{H}^{1}(0,1)$ and $\mathrm{L}^{2}(\Omega)$, respectively, where $d$ and $n$ are the discretization parameters. In the spatial variable we have used a single Gauss-Lobatto-Legendre spectral finite element $[21,29]$ of degree $d=20$. Since the solution $u$ is smooth in $x$ the spectral element method converges extremely fast with increasing $d$, allowing us to essentially eliminate the discretization error with respect to the spatial variable $x$. Denoting by $\left\{p_{j}\right\}_{j=0}^{n}$ the orthogonal polynomials used in the chaos expansion, and by $\left\{\phi_{k}\right\}_{k=1}^{d}$ the Lagrange basis of the spectral element, the stochastic Galerkin trial and test functions have the form

$$
u_{d, n}(x, \xi)=\sum_{j=0}^{n} \sum_{k=1}^{d} u_{j, k} \phi_{k}(x) p_{j}(\xi), \quad u_{j, k} \in \mathbb{R} .
$$

Constructing the stochastic Galerkin equations requires first a chaos expansion of the input random field $a$. For the Hermite chaos the expansion coefficients are given by

$$
a_{k}(x)=\left\langle a(x, \omega) h_{k}(\xi(\omega)\rangle=\int_{-\infty}^{\infty} \mathrm{e}^{|\xi| x} h_{k}(\xi) \frac{1}{\sqrt{2 \pi}} \mathrm{e}^{\frac{-\xi^{2}}{2}} \mathrm{~d} \xi,\right.
$$

with $h_{k}$ is the normalized Hermite polynomial of degree $k$. These can be obtained in closed form as

$$
a_{2 m}(x)=\frac{1}{\sqrt{(2 m) !}}\left(2 x^{2 m} F_{\xi}(x) \mathrm{e}^{\frac{x^{2}}{2}}+\sqrt{\frac{2}{\pi}} x \sum_{i=1}^{m}(-1)^{i-1} x^{2(m-i)}(2 i-3) ! !\right)
$$

when $k=2 m, m \in \mathbb{N}_{0}$, and $a_{k}=0$ for odd $k$. Here $F_{\xi}$ denotes the standard Gaussian probability distribution function and $n$ !! the double factorial. For details as well as a derivation of (4.10) we refer to Appendix C.

For the generalized polynomial chaos with respect to the reflected Gaussian distribution many required quantities are not available in closed form, among these the orthogonal polynomials themselves and the expansion coefficients of the input random field. The polynomials can be constructed using the Stieltjes process once their three-term recurrence coefficients have been computed. A general computational technique for this can be found in the book by Gautschi [13]. Moreover, the recurrence coefficients permit the construction of Gauss quadrature rules adapted to the reflected Gaussian density which then allow the computation of, e.g., the generalized chaos coefficients of the input random field as well as other related integrals.

Figure 1 shows the relative error in the second moment over the spatial domain of a stochastic Galerkin approximation to the solution of (4.7) using standard Hermite chaos approximations in $\xi$ of degrees $n=5,10,15$ and 20 compared to generalized polynomial chaos with respect to the reflected Gaussian random variable $|\xi|$ of degrees $n=2$ and 5 . It is apparent that the approximation based on the generalized polynomial chaos expansion 


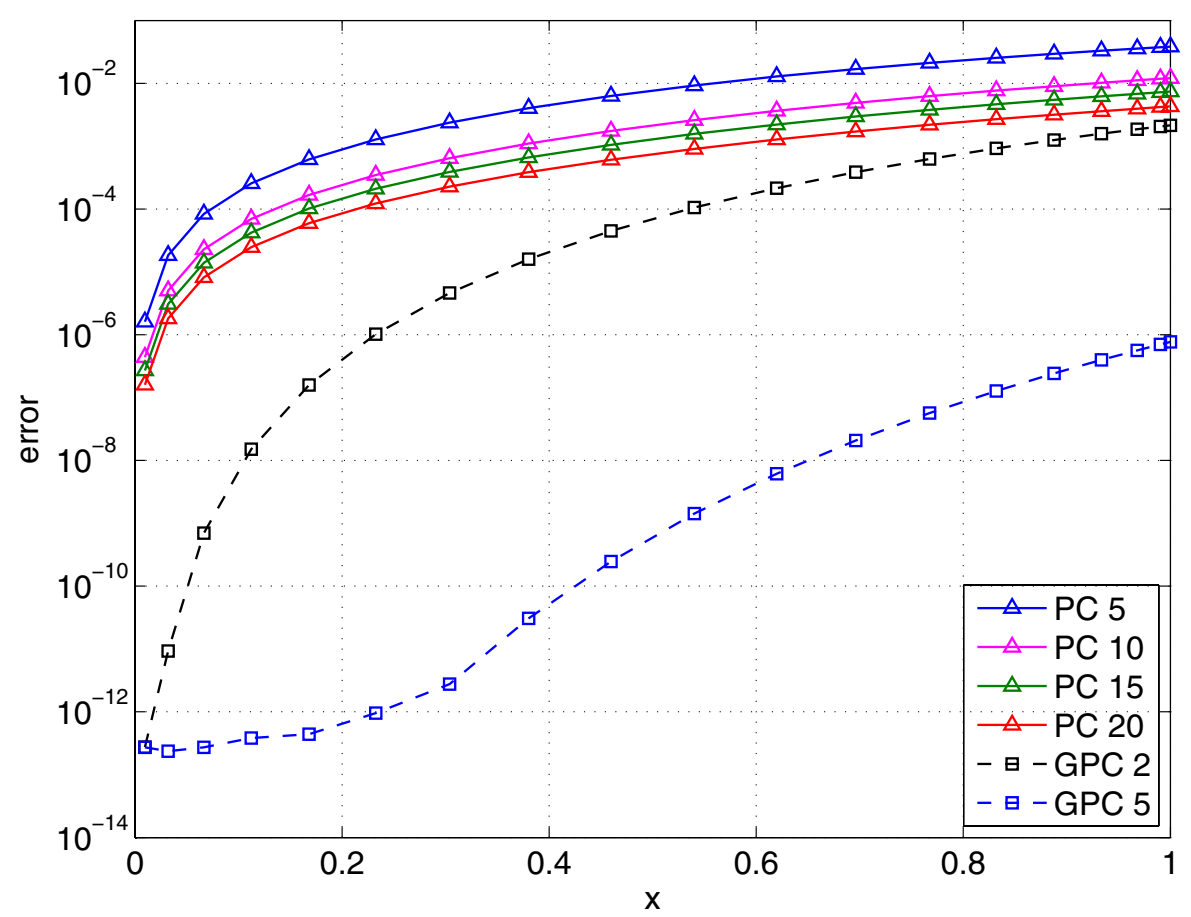

FIGURE 1. (Pointwise) relative error of second moment of the stochastic Galerkin approximation to the solution of (4.7) with $f \equiv 1, F=1$ and random field $a(x, \omega)=\exp (|\xi(\omega)| x)$ using standard (PC) and generalized (GPC) polynomial chaos expansions of various orders in the stochastic variables. The markers indicate the locations of the Gauss-Lobatto-Legendre nodes used in the spectral element discretization in $x$.

gives a better approximation with lower polynomial degrees. Similar results are obtained for the error in the mean (first moment). Table 1 gives the relative error in the first and second moments

$$
\frac{\left\|\left\langle u(x, \cdot)^{k}\right\rangle-\left\langle u_{d, n}(x, \cdot)^{k}\right\rangle\right\|}{\left\|\left\langle u(x, \cdot)^{k}\right\rangle\right\|} \quad k=1,2,
$$

in $\mathrm{L}^{2}$ - and $\mathrm{H}^{1}$-norms for both approximation types using $d=24$ in the spatial discretization and polynomial degrees $n=2,5,8$ and 10 in the stochastic variable. Again the much higher convergence rate of the generalized polynomial chaos discretization is evident.

This example clearly demonstrates the possible benefits of generalized polynomial chaos expansions in stochastic Galerkin approximations over standard Wiener-Hermite chaos expansions. By using chaos polynomials taylored to the particular probabilistic setting/basic random variables a much faster convergence of the Galerkin approximation can be achieved. Bearing in mind the lognormal random variables as an example, we have, however, also demonstrated that a careful study of the basic random variables is necessary to ensure the convergence of generalized polynomial chaos expansions to the desired limit.

\section{Summary}

We have reviewed the constructions of standard as well as generalized polynomial chaos expansions of random variables with finite second moments, and we have shown under what conditions the results of the Cameron 
TABLE 1. Relative $\mathrm{L}^{2}$ - and $\mathrm{H}^{1}$-norm errors of the first and second moments in the stochastic Galerkin approximation of to the solution of (4.7) with $f \equiv 1, F=1$ and random field $a(x, \omega)=\exp (|\xi(\omega)| x)$ using standard (PC) and generalized (GPC) polynomial chaos expansions of various orders in the stochastic variables.

\begin{tabular}{|c|c|c|c|c|c|}
\hline$n$ & Chaos type & $\mathrm{L}^{2}$ error $\langle u\rangle$ & $\mathrm{H}^{1}$ error $\langle u\rangle$ & $\mathrm{L}^{2}$ error $\left\langle u^{2}\right\rangle$ & $\mathrm{H}^{1}$ error $\left\langle u^{2}\right\rangle$ \\
\hline 5 & PC & $7.2 \mathrm{e}-03$ & $1.4 \mathrm{e}-02$ & $2.5 \mathrm{e}-02$ & $4.8 \mathrm{e}-02$ \\
10 & PC & $2.0 \mathrm{e}-03$ & $4.0 \mathrm{e}-03$ & $7.8 \mathrm{e}-03$ & $1.5 \mathrm{e}-02$ \\
15 & PC & $1.2 \mathrm{e}-03$ & $2.4 \mathrm{e}-03$ & $4.7 \mathrm{e}-03$ & $2.4 \mathrm{e}-03$ \\
20 & PC & $6.9 \mathrm{e}-04$ & $1.4 \mathrm{e}-03$ & $2.7 \mathrm{e}-03$ & $5.4 \mathrm{e}-03$ \\
\hline 2 & GPC & $1.9 \mathrm{e}-04$ & $6.1 \mathrm{e}-04$ & $1.1 \mathrm{e}-03$ & $3.3 \mathrm{e}-03$ \\
5 & GPC & $1.6 \mathrm{e}-08$ & $1.0 \mathrm{e}-07$ & $3.0 \mathrm{e}-07$ & $1.6 \mathrm{e}-06$ \\
8 & GPC & $5.0 \mathrm{e}-13$ & $5.3 \mathrm{e}-12$ & $3.5 \mathrm{e}-11$ & $2.7 \mathrm{e}-10$ \\
10 & GPC & $1.4 \mathrm{e}-14$ & $5.6 \mathrm{e}-13$ & $6.6 \mathrm{e}-14$ & $1.5 \mathrm{e}-12$ \\
\hline
\end{tabular}

Martin theorem extend from standard to generalized polynomial chaos expansions with specific analysis of expansions in one, finitely many and countably many random variables. This closes a gap in the theory of generalized polynomial chaos expansions. Finally, we have presented examples illustrating non-approximability by generalized polynomial chaos expansions as well as accelerated convergence compared to standard polynomial chaos expansion in the context of a stochastic Galerkin approximation.

\section{Appendix A. The orthonormal POLYNOMials FOR A LOGNORMAl DENSITY}

The Stieltjes-Wigert polynomials (cf. [41], Sect. 2.7 and [8], Chap. VI, Sect. 2) are orthonormal with respect to the family of weight functions

$$
w_{\nu}(x)=\left\{\begin{array}{ll}
\frac{\nu}{\sqrt{\pi}} \mathrm{e}^{-\nu^{2} \log ^{2} x}, & x>0, \\
0, & \text { otherwise }
\end{array} \quad \nu>0\right.
$$

and are given by

$$
q_{k}(x)=(-1)^{k} a^{(2 k+1) / 4}[a]_{k}^{-1 / 2} \sum_{j=0}^{k}\left[\begin{array}{l}
k \\
j
\end{array}\right]_{a} a^{j^{2}}\left(-a^{1 / 2} x\right)^{j}, \quad k \geq 0,
$$

where $a=\exp \left(-1 /\left(2 \nu^{2}\right)\right)$ and we have introduced the notation

$$
[a]_{0}=1, \quad[a]_{k}=\left(1-a^{k}\right)\left(1-a^{k-1}\right) \cdots(1-a), \quad k \geq 1,
$$

as well as the generalized binomial coefficient or Gauss symbol

$$
\left[\begin{array}{c}
k \\
j
\end{array}\right]_{a}=\frac{[a]_{k}}{[a]_{k-j}[a]_{j}}=\frac{\left(1-a^{k}\right)\left(1-a^{k-1}\right) \cdots\left(1-a^{k-j+1}\right)}{\left(1-a^{j}\right)\left(1-a^{j-1}\right) \cdots(1-a)}, \quad\left[\begin{array}{c}
k \\
0
\end{array}\right]_{a}=\left[\begin{array}{l}
k \\
k
\end{array}\right]_{a}=1
$$

We proceed to construct from these the orthonormal polynomials associated with the lognormal probability density function

$$
f(x)= \begin{cases}\frac{1}{x \sqrt{2 \pi}} \mathrm{e}^{-\frac{1}{2} \log ^{2} x}, & x>0 \\ 0, & x \leq 0 .\end{cases}
$$


Proposition A.1. The polynomials $\left\{p_{k}\right\}_{k \in \mathbb{N}_{0}}$ orthonormal with respect to the lognormal density (A.2) are given by

$$
p_{0}(x) \equiv 1, \quad p_{k}(x)=\frac{(-1)^{k} \mathrm{e}^{k(k-1) / 4}}{\sqrt{\prod_{i=1}^{k}\left(\mathrm{e}^{i}-1\right)}} \sum_{j=0}^{k}(-1)^{j}\left[\begin{array}{c}
k \\
j
\end{array}\right]_{a} \mathrm{e}^{-j^{2}+j / 2} x^{j}, \quad k \geq 1,
$$

with $a=1 / \mathrm{e}$.

Proof. We denote by $\left\{\widetilde{q}_{k}\right\}_{k \in \mathbb{N}_{0}}$ the particular sequence of Stieltjes-Wigert polynomials obtained for the parameter value $\nu=1 / \sqrt{2}$ with associated weight function

$$
\widetilde{w}(x)= \begin{cases}\frac{1}{\sqrt{2 \pi}} \mathrm{e}^{-\frac{1}{2} \log ^{2} x}, & x>0 \\ 0, & \text { otherwise }\end{cases}
$$

In view of

$$
\mathrm{e}^{1 / 4} \widetilde{q}_{0}(\mathrm{e} x)=\mathrm{e}^{1 / 4} \mathrm{e}^{-1 / 4}=1
$$

as well as

$$
\begin{aligned}
\mathrm{e}^{1 / 4} \widetilde{q}_{k}(\mathrm{e} x) & =\frac{(-1)^{k} \mathrm{e}^{-k / 2}}{\sqrt{\prod_{i=1}^{k}\left(1-\mathrm{e}^{-i}\right)}} \sum_{j=0}^{k}\left[\begin{array}{c}
k \\
j
\end{array}\right]_{a} \mathrm{e}^{-j^{2}}\left(-\mathrm{e}^{-1 / 2} \mathrm{e} x\right)^{j} \\
& =\frac{(-1)^{k} \mathrm{e}^{-k / 2+k(k+1) / 4}}{\sqrt{\prod_{i=1}^{k}\left(\mathrm{e}^{i}-1\right)}} \sum_{j=0}^{k}\left[\begin{array}{c}
k \\
j
\end{array}\right]_{a}(-1)^{j} \mathrm{e}^{-j^{2}+j / 2} x^{j} \\
& =\frac{(-1)^{k} \mathrm{e}^{k(k-1) / 4}}{\sqrt{\prod_{i=1}^{k}\left(\mathrm{e}^{i}-1\right)}} \sum_{j=0}^{k}\left[\begin{array}{c}
k \\
j
\end{array}\right]_{a}(-1)^{j} \mathrm{e}^{-j^{2}+j / 2} x^{j}, \quad k \geq 1,
\end{aligned}
$$

we obtain the relation

$$
p_{k}(x)=\mathrm{e}^{1 / 4} \widetilde{q}_{k}(\mathrm{e} x), \quad k \in \mathbb{N}_{0} .
$$

Orthonormality now follows after a succession of changes of variables from

$$
\begin{aligned}
& \int_{0}^{\infty} p_{k}(x) p_{\ell}(x) f(x) \mathrm{d} x=\int_{0}^{\infty} \mathrm{e}^{1 / 4} \widetilde{q}_{k}(\mathrm{e} x) \mathrm{e}^{1 / 4} \widetilde{q}_{\ell}(\mathrm{e} x) f(x) \mathrm{d} x \\
& \quad=\sqrt{\frac{e}{2 \pi}} \int_{-\infty}^{\infty} \widetilde{q}_{k}\left(\mathrm{e}^{y+1}\right) \widetilde{q}_{\ell}\left(\mathrm{e}^{y+1}\right) \mathrm{e}^{-\frac{1}{2} y^{2}} \mathrm{~d} y=\int_{-\infty}^{\infty} \widetilde{q}_{k}\left(\mathrm{e}^{z}\right) \widetilde{q}_{\ell}\left(\mathrm{e}^{z}\right) \frac{\mathrm{e}^{-\frac{1}{2} z^{2}} \mathrm{e}^{z}}{\sqrt{2 \pi}} \mathrm{d} z \\
& =\int_{0}^{\infty} \widetilde{q}_{k}(t) \widetilde{q}_{\ell}(t) \frac{\mathrm{e}^{-\frac{1}{2} \log ^{2} t}}{\sqrt{2 \pi}} \mathrm{d} t=\int_{0}^{\infty} \widetilde{q}_{k}(t) \widetilde{q}_{\ell}(t) \widetilde{w}(t) \mathrm{d} t=\delta_{k \ell} .
\end{aligned}
$$

Like all orthogonal polynomials over the real numbers, the polynomials $\left\{p_{k}\right\}_{k \in \mathbb{N}}$ satisfy a three-term recurrence relation

$$
\sqrt{\beta_{k+1}} p_{k+1}(x)=\left(x-\alpha_{k}\right) p_{k}(x)-\sqrt{\beta_{k}} p_{k-1}(x), \quad k \geq 0,
$$


with $p_{-1} \equiv 0$ and $p_{0} \equiv 1$, where we follow the common convention of denoting by $\left\{\alpha_{k}\right\}_{k \in \mathbb{N}_{0}}$ and $\left\{\beta_{k}\right\}_{k \in \mathbb{N}_{0}}$ the recurrence coefficients of the associated monic orthogonal polynomials (cf. [13], Sect. 1.3). Since the weight function $f$ of the $\left\{p_{k}\right\}$ is a probability density function we must have

$$
\beta_{0}=\int_{0}^{\infty} p_{0}(x)^{2} f(x) \mathrm{d} x=1 .
$$

The remaining coefficients are obtained from the explicit representation (A.3). If we denote the $j$-th polynomial coefficient of $p_{k}$ by $c_{j}^{(k)}$, i.e., such that

$$
p_{k}(x)=\sum_{j=0}^{k} c_{j}^{(k)} x^{j}, \quad k \in \mathbb{N}_{0},
$$

then by (A.3) we have

$$
c_{j}^{(k)}=\frac{(-1)^{k+j} \mathrm{e}^{k(k-1) / 4}}{\sqrt{\prod_{i=1}^{k}\left(\mathrm{e}^{i}-1\right)}}\left[\begin{array}{l}
k \\
j
\end{array}\right]_{a} \mathrm{e}^{-j^{2}+j / 2}, \quad j=0, \ldots, k, \quad k \in \mathbb{N}_{0} .
$$

Comparing coefficients in (A.4) taking account of $p_{-1} \equiv 0$ and $p_{0} \equiv 1$, we find

$$
\beta_{1}=\left(\frac{1}{c_{1}^{(1)}}\right)^{2}, \quad \alpha_{0}=-\frac{c_{0}^{(1)}}{c_{1}^{(1)}}
$$

and, in general,

$$
\beta_{k+1}=\left(\frac{c_{k}^{(k)}}{c_{k+1}^{(k+1)}}\right)^{2}, \quad \alpha_{k}=\frac{c_{k-1}^{(k)}}{c_{k}^{(k)}}-\frac{c_{k}^{(k+1)}}{c_{k+1}^{(k+1)}}, \quad k \in \mathbb{N} .
$$

Together with (A.5), a straightforward calculation yields

$$
\alpha_{k}=\mathrm{e}^{k-1 / 2}\left(\mathrm{e}^{k}(\mathrm{e}+1)-1\right), \quad \beta_{k+1}=\left(\mathrm{e}^{k+1}-1\right) \mathrm{e}^{3 k+1}, \quad k \in \mathbb{N}_{0} .
$$

\section{Appendix B. Lognormal ChaOs COEFFicients of THE RECIPROCAL of A LOGNORMAL RANDOM VARIABLE}

Proposition B.1. The generalized polynomial chaos coefficients $\left\{a_{k}\right\}_{k \in \mathbb{N}_{0}}$ of the random variable $\zeta$ defined in (4.5) with respect to the orthonormal polynomials $\left\{p_{k}\right\}_{k \in \mathbb{N}_{0}}$ associated with the lognormal density and defined in (4.4) in $\eta$ are given by

$$
a_{0}=\mathrm{e}^{1 / 2}, \quad a_{k}=(-1)^{k} \mathrm{e}^{-\left(k^{2}+3 k-2\right) / 4} \sqrt{\prod_{i=1}^{k}\left(\mathrm{e}^{i}-1\right)}, \quad k \geq 1 .
$$

Proof. The first coefficient $a_{0}$ of $\zeta$ is obtained as

$$
\begin{aligned}
a_{0} & =\left\langle\zeta p_{0}(\eta)\right\rangle=\int_{0}^{\infty} \frac{1}{x} \cdot 1 \cdot f_{\eta}(x) \mathrm{d} x=\int_{0}^{\infty} \frac{\mathrm{e}^{-\frac{1}{2} \log ^{2} x}}{x^{2} \sqrt{2 \pi}} \mathrm{d} x \\
& =\frac{1}{\sqrt{2 \pi}} \int_{-\infty}^{\infty} \mathrm{e}^{-y} \mathrm{e}^{-\frac{1}{2} y^{2}} \mathrm{~d} y=\sqrt{\mathrm{e}} .
\end{aligned}
$$


The remaining coefficients $a_{k}$ are obtained by induction making use of the recurrence (4.4). For $k=1$ this results in

$$
a_{1}=\left\langle\zeta p_{1}(\eta)\right\rangle=\left\langle\frac{1}{\eta} \frac{\eta-\alpha_{0}}{\sqrt{\beta_{1}}}\right\rangle=\frac{1}{\sqrt{\beta_{1}}}-\frac{\alpha_{0}}{\sqrt{\beta_{1}}}\left\langle\frac{1}{\eta}\right\rangle=-\mathrm{e}^{-1 / 2} \sqrt{\mathrm{e}-1},
$$

in agreement with (B.1). Assuming (B.1) holds for all $0 \leq j \leq k$, we obtain from the recurrence relation (4.4)

$$
\begin{aligned}
a_{k+1} & =\left\langle\zeta p_{k+1}(\eta)\right\rangle=\left\langle\frac{\left(\eta-\alpha_{k}\right) p_{k}(\eta)-\sqrt{\beta_{k}} p_{k-1}(\eta)}{\eta \sqrt{\beta_{k+1}}}\right\rangle=-\frac{\alpha_{k} a_{k}+\sqrt{\beta_{k}} a_{k-1}}{\sqrt{\beta_{k+1}}} \\
& =-\frac{\left(\mathrm{e}^{k / 2}+\mathrm{e}^{k / 2-1}-\mathrm{e}^{-k / 2-1}\right) a_{k}+\sqrt{\mathrm{e}^{k}-1} \mathrm{e}^{-3 / 2} a_{k-1}}{\sqrt{\mathrm{e}^{k+1}-1}} \\
& =(-1)^{k+1} \mathrm{e}^{-\left(k^{2}+3 k-2\right) / 4} \sqrt{\prod_{i=1}^{k}\left(\mathrm{e}^{i}-1\right)} \frac{\mathrm{e}^{k / 2}-\mathrm{e}^{-k / 2-1}}{\sqrt{\mathrm{e}^{k+1}-1}} \\
& =(-1)^{k+1} \mathrm{e}^{-\left(k^{2}+3 k-2\right) / 4} \sqrt{\prod_{i=1}^{k}\left(\mathrm{e}^{i}-1\right) \mathrm{e}^{-k / 2-1} \frac{\mathrm{e}^{k+1}-1}{\sqrt{\mathrm{e}^{k+1}-1}}} \\
& =(-1)^{k+1} \mathrm{e}^{-\left((k+1)^{2}+3(k+1)-2\right) / 4} \sqrt{\prod_{i=1}^{k+1}\left(\mathrm{e}^{i}-1\right)} .
\end{aligned}
$$

\section{Appendix C. Hermite Chaos coefficients of $\mathrm{e}^{|\xi| x}$}

In this section we give a derivation of the Hermite chaos coefficients of the random field (4.8) in Section 4.3.

Proposition C.1. The coefficients (4.9) in the Hermite polynomial chaos expansion of the random field (4.8) are given by

$$
a_{2 m}(x)=\frac{1}{\sqrt{(2 m) !}}\left(2 x^{2 m} F_{\xi}(x) \mathrm{e}^{\frac{x^{2}}{2}}+\sqrt{\frac{2}{\pi}} x \sum_{i=1}^{m}(-1)^{i-1} x^{2(m-i)}(2 i-3) ! !\right), m \in \mathbb{N}_{0},
$$

where $F_{\xi}(x)=\frac{1}{\sqrt{2 \pi}} \int_{-\infty}^{x} \mathrm{e}^{-\frac{y^{2}}{2}} \mathrm{~d} y$ denotes the standard Gaussian probability distribution function and $n ! !$ the double factorial defined for integers $n \geq-1$ by

$$
n ! !:= \begin{cases}n(n-2) \cdots 3 \cdot 1, & n \text { odd } \\ n(n-2) \cdots 4 \cdot 2, & n \text { even } \\ 1, & n=0,-1\end{cases}
$$

Proof. We note first that, since $e^{|\xi| x}$ is an even function of $\xi$, its odd Hermite chaos coefficients vanish. For $m=0$ we obtain

$$
a_{0}(x)=\frac{2}{\sqrt{2 \pi}} \int_{0}^{\infty} \mathrm{e}^{\xi x} \mathrm{e}^{\frac{-\xi^{2}}{2}} \mathrm{~d} \xi=\sqrt{\frac{2}{\pi}} \mathrm{e}^{\frac{x^{2}}{2}} \int_{0}^{\infty} \mathrm{e}^{-\frac{(\xi-x)^{2}}{2}} \mathrm{~d} \xi=2 F_{\xi}(x) \mathrm{e}^{\frac{x^{2}}{2}}
$$


Proceeding by induction and noting that the even Hermite polynomials are even functions, we obtain for $m \geq 0$

$$
\begin{aligned}
a_{2 m+2}(x) & =\sqrt{\frac{2}{\pi}} \int_{0}^{\infty} \mathrm{e}^{\xi x} h_{2 m+2}(\xi) \mathrm{e}^{-\frac{\xi^{2}}{2}} \mathrm{~d} \xi \\
& =\sqrt{\frac{2}{\pi}} \int_{0}^{\infty} \mathrm{e}^{\xi x} \frac{(-1)^{2 m+2}}{\sqrt{(2 m+2) !}}\left(\frac{d^{2 m+2}}{\mathrm{~d} \xi^{2 m+2}} \mathrm{e}^{-\frac{\xi^{2}}{2}}\right) \mathrm{d} \xi
\end{aligned}
$$

where we have used the Rodrigues' formula (4.3) to express the normalized Hermite polynomials $h_{k}$. Integrating twice by parts gives

$$
\begin{aligned}
& a_{2 m+2}(x)=\sqrt{\frac{2}{\pi(2 m+2) !}}\left[x H_{2 m}(0)+x^{2} \int_{0}^{\infty} \mathrm{e}^{\xi x}\left(\frac{\mathrm{d}^{2 m}}{\mathrm{~d} \xi^{2 m}} \mathrm{e}^{-\frac{\xi^{2}}{2}}\right) \mathrm{d} \xi\right] \\
& =\sqrt{\frac{2}{\pi(2 m+2) !}}\left[x H_{2 m}(0)+x^{2} \int_{0}^{\infty} \mathrm{e}^{\xi x} H_{2 m}(\xi) \mathrm{e}^{-\frac{\xi^{2}}{2}} \mathrm{~d} \xi\right] \\
& =x \sqrt{\frac{2}{\pi(2 m+2) !}} H_{2 m}(0)+x^{2} \sqrt{\frac{(2 m) !}{(2 m+2) !}} \sqrt{\frac{2}{\pi}} \int_{0}^{\infty} \mathrm{e}^{\xi x} h_{2 m}(\xi) \mathrm{e}^{-\frac{\xi^{2}}{2}} \mathrm{~d} \xi \\
& =\frac{1}{\sqrt{(2 m+2) !}}\left(x \sqrt{\frac{2}{\pi}}(-1)^{m}(2 m-1) ! !+x^{2} \sqrt{(2 m) !} a_{2 m}(x)\right),
\end{aligned}
$$

whereupon the assertion follows by inserting (C.1) for $a_{2 m}(x)$.

\section{REFERENCES}

[1] M. Arnst, R. Ghanem and C. Soize, Identification of Bayesian posteriors for coefficients of chaos expansions. J. Comput. Phys. 229 (2010) 3134-3154.

[2] I. Babuška, R. Tempone and G.E. Zouraris, Galerkin finite element approximations of stochastic elliptic partial differential equations. SIAM J. Numer. Anal. 42 (2004) 800-825.

[3] I. Babuška, R. Tempone and G.E. Zouraris, Solving elliptic boundary value problems with uncertain coefficients by the finite element method: The stochastic formulation. Comput. Methods Appl. Mech. Engrg. 194 (2005) 1251-1294.

[4] C. Berg, Moment problems and polynomial approximation. Ann. Fac. Sci. Toulouse Math. (Numéro spécial Stieltjes) 6 (1996) 9-32.

[5] C. Berg and J.P.R. Christensen, Density questions in the classical theory of moments. Ann. Inst. Fourier 31 (1981) 99-114.

[6] A. Bobrowski, Functional Analysis for Probability and Stochastic Processes. Cambridge University Press, Cambridge UK (2005).

[7] R.H. Cameron and W.T. Martin, The orthogonal development of non-linear functionals in series of Fourier-Hermite functionals. Ann. Math. 48 (1947) 385-392.

[8] T.S. Chihara, An Introduction to Orthogonal Polynomials. Gordon and Breach, New York (1978).

[9] J.H. Curtiss, A note on the theory of moment generating functions. Ann. Stat. 13 (1942) 430-433.

[10] B.J. Debusschere, H.N. Najm, Ph.P. Pébay, O.M. Knio, R.G. Ghanem and O.P. le Maître, Numerical challenges in the use of polynomial chaos representations for stochastic processes. SIAM J. Sci. Comput. 26 (2004) 698-719.

[11] R.V. Field Jr. and M. Grigoriu, On the accuracy of the polynomial chaos expansion. Probab. Engrg. Mech. 19 (2004) 65-80.

[12] G. Freud, Orthogonal Polynomials. Akademiai, Budapest (1971).

[13] W. Gautschi, Orthogonal Polynomials: Computation and Approximation. Oxford University Press (2004).

[14] R. Ghanem and P.D. Spanos, Stochastic Finite Elements: A Spectral Approach. Springer-Verlag, New York (1991).

[15] A. Gut, On the moment problem. Bernoulli 8 (2002) 407-421.

[16] T. Hida, Brownian Motion. Springer, New York (1980).

[17] K. Itô, Multiple Wiener integral. J. Math. Soc. Jpn 3 (1951) 157-169.

[18] S. Janson, Gaussian Hilbert Spaces. Cambridge University Press, Cambridge (1997).

[19] O. Kallenberg, Foundations of Modern Probability, 2nd edition. Springer-Verlag, New York (2002).

[20] G. Kallianpur, Stochastic Filtering Theory. Springer, New York (1980).

[21] G.E. Karniadakis and S. Sherwin, Spectral/hp Element Methods for Computational Fluid Dynamics, 2nd edition. Oxford University Press (2005). 
[22] G.E. Karniadakis, C.-H. Shu, D. Xiu, D. Lucor, C. Schwab and R.-A. Todor, Generalized polynomial chaos solution for differential equations with random inputs. Technical Report 2005-1, Seminar for Applied Mathematics, ETH Zürich, Zürich, Switzerland (2005).

[23] A.N. Kolmogorov, Grundbegriffe der Wahrscheinlichkeitsrechnung. Springer, Berlin (1933).

[24] G.D. Lin, On the moment problems. Stat. Probab. Lett. 35 (1997) 85-90. Correction: G.D. Lin, On the moment problems. Stat. Probab. Lett. 50 (2000) 205.

[25] P. Masani, Wiener's contributions to generalized harmonic analysis, prediction theory and filter theory. Bull. Amer. Math. Soc. 72 (1966) 73-125.

[26] P.R. Masani, Norbert Wiener, 1894-1964. Number 5 in Vita mathematica, Birkhäuser (1990).

[27] H.G. Matthies and C. Bucher, Finite elements for stochastic media problems. Comput. Methods Appl. Mech. Engrg. 168 (1999) 3-17.

[28] A. Mugler and H.-J. Starkloff, On elliptic partial differential equations with random coefficients, Stud. Univ. Babes-Bolyai Math. 56 (2011) 473-487.

[29] A.T. Patera, A spectral element method for fluid dynamics - laminar flow in a channel expansion. J. Comput. Phys. 54 (1984) $468-488$.

[30] R.E.A.C. Payley and N. Wiener, Fourier Transforms in the Complex Domain. Number XIX in Colloquium Publications. Amer. Math. Soc. (1934).

[31] L.C. Petersen, On the relation between the multidimensional moment problem and the one-dimensional moment problem. Math. Scand. 51 (1982) 361-366.

[32] M. Reed and B. Simon, Methods of modern mathematical physics, Functional analysis 1. Academic press, New York (1972).

[33] M. Riesz, Sur le problème des moments et le théorème de Parseval correspondant. Acta Litt. Ac. Scient. Univ. Hung. 1 (1923) 209-225.

[34] R.A. Roybal, A reproducing kernel condition for indeterminacy in the multidimensional moment problem. Proc. Amer. Math. Soc. 135 (2007) 3967-3975.

[35] I.E. Segal, Tensor algebras over Hilbert spaces. I, Trans. Amer. Math. Soc. 81 (1956) 106-134.

[36] A.N. Shiryaev, Probability. Springer-Verlag, New York (1996).

[37] I.C. Simpson, Numerical integration over a semi-infinite interval using the lognormal distribution. Numer. Math. 31 (1978) $71-76$.

[38] C. Soize and R. Ghanem, Physical systems with random uncertainties: Chaos representations with arbitrary probability measures. SIAM J. Sci. Comput. 26 (2004) 395-410.

[39] H.-J. Starkloff, On the number of independent basic random variables for the approximate solution of random equations, in Celebration of Prof. Dr. Wilfried Grecksch's 60th Birthday, edited by C. Tammer and F. Heyde. Shaker Verlag, Aachen (2008) 195-211.

[40] J.M. Stoyanov, Counterexamples in Probability, 2nd edition. John Wiley \& Sons Ltd., Chichester, UK (1997).

[41] G. Szegö, Orthogonal Polynomials. American Mathematical Society, Providence, Rhode Island (1939).

[42] R.-A. Todor and C. Schwab, Convergence rates for sparse chaos approximations of elliptic problems with stochastic coefficients. IMA J. Numer. Anal. 27 (2007) 232-261.

[43] N. Wiener, Differential space. J. Math. Phys. 2 (1923) 131-174.

[44] N. Wiener, Generalized harmonic analysis. Acta Math. 55 (1930) 117-258.

[45] N. Wiener, The homogeneous chaos. Amer. J. Math. 60 (1938) 897-936.

[46] D. Xiu and J.S. Hesthaven, High-order collocation methods for differential equations with random inputs. SIAM J. Sci. Comput. 27 (2005) 1118-1139.

[47] D. Xiu and G.E. Karniadakis, Modeling uncertainty in steady state diffusion problems via generalized polynomial chaos. Comput. Methods Appl. Mech. Engrg. 191 (2002) 4927-4948.

[48] D. Xiu and G.E. Karniadakis, The Wiener-Askey polynomial chaos for stochastic differential equations. SIAM J. Sci. Comput. 24 (2002) 619-644.

[49] D. Xiu and G.E. Karniadakis, A new stochastic approach to transient heat conduction modeling with uncertainty. Int. J. Heat Mass Trans. 46 (2003) 4681-4693.

[50] D. Xiu and G.E. Karniadakis, Modeling uncertainty in flow simulations via generalized polynomial chaos. J. Comput. Phy. 187 (2003) 137-167.

[51] D. Xiu, D. Lucor, C.-H. Su and G.E. Karniadakis, Stochastic modeling of flow-structure interactions using generalized polynomial chaos. J. Fluids Eng. 124 (2002) 51-59.

[52] D. Xiu, D. Lucor, C.-H. Su and G.E. Karniadakis, Performance evaluation of generalized polynomial chaos, in Computational Science - ICCS 2003, Lecture Notes in Computer Science 2660, edited by P.M.A. Sloot, D. Abramson, A.V. Bogdanov, J.J. Dongarra, A.Y. Zomaya and Y.E. Gorbachev. Springer-Verlag (2003).

[53] Y. Xu, On orthogonal polynomials in several variables, in Special functions, q-series, and related topics, edited by M. Ismail, D.R. Masson and M. Rahman. Fields Institute Communications 14 (1997) 247-270. 